\title{
Statical and dynamical behaviour of thin fibre reinforced composite laminates with different shapes
}

\author{
Liz Graciela Nallim $^{\mathrm{a}, *}$, Sergio Oller Martinez ${ }^{\mathrm{b}}$, Ricardo Oscar Grossi ${ }^{\text {a }}$ \\ a CONICET, ICMASA, Facultad de Ingeniería, Universidad Nacional De Salta, Av. Bolivia 5150, 4400 Salta, Argentina \\ ${ }^{\mathrm{b}}$ Departamento de Resistencia de Materiales y Estructuras en la Ingeniería, Universidad Politécnica de Cataluña, \\ Campus Norte UPC, Gran Capitán S/N, 08034 Barcelona, Spain
}

Received 19 April 2004; received in revised form 24 June 2004; accepted 25 June 2004

\begin{abstract}
Based on the classical laminated plate theory, a variational approach for the study of the statical and dynamical behaviour of arbitrary quadrilateral anisotropic plates with various boundary conditions is developed. The analytical formulation uses the Ritz method in conjunction with natural coordinates to express the geometry of general plates in a simple form. The deflection of the plate is approximated by a set of beam characteristic orthogonal polynomials generated using the Gram-Schmidt procedure. The algorithm developed is quite general and can be used to study fibre reinforced composite laminates with symmetric lay-ups, which may have general anisotropy and any combinations of clamped, simply supported and free edge support conditions. Various numerical applications are presented and some results are compared with existing values in the literature to demonstrate the accuracy and flexibility of the present method. New results were also determined for plates with different geometrical shapes, combinations of boundary conditions, several stacking sequences and various angles of fibre orientation.
\end{abstract}

(C) 2004 Elsevier B.V. All rights reserved.

Keywords: Composite plates; Laminates; Ritz method; Free vibration; Mode shapes; Static analysis

\section{Introduction}

Composite structures, especially laminated composite plates, have been widely used in many engineering advantages of high strength (as well as high stiffness) and light weight. Another advantage of the laminated composite plate is the controllability of the structural properties through changing the fibre orientation

\footnotetext{
${ }^{*}$ Corresponding author. Fax: +54 03874255351.

E-mail address: lnallim@unsa.edu.ar (L.G. Nallim).
} 
angles, the number of plies and selecting proper composite materials. With the wide use of composite plate structures in modern industries, dynamic and static analysis of plates of complex geometry becomes an important design procedure. An adequate understanding of the free vibration and the flexural behaviour of these plates components, is crucial to the design and performance evaluation of a mechanical system. However, static and dynamic solutions to these plate problems are strongly dependent on the geometrical shape, boundary conditions and material properties. It is widely recognised that closed form solutions are possible only for a few specific cases [1,2].

Analytical studies about vibration of isotropic and anisotropic plates of different shapes and configurations are well documented. The excellent reviews of Leissa [2,3], Blevins [4] and Bert [5-7], show that most of these results are for isotropic and orthotropic rectangular, circular, elliptical and other regular shaped plates. Nevertheless, analytical studies on general quadrilateral laminated plates with unequal side lengths and different combinations of boundary conditions are rather limited. This may be due to the difficulty in forming a simple and adequate deflection function which can be applied to the entire plate domain and satisfy the boundary conditions. In general, for the analysis of arbitrary shaped plates, several numerical techniques such as finite elements, finite difference and finite strip methods have been deployed by many researchers (see for instance Refs. [8-13]). Although the discretisation methods provide a general framework for the analysis of general plates, they invariably result in problems which possess a large number of degrees of freedom. Therefore, for large scale structural design and analysis, where repeated calculations are often required, one may think of using the Ritz method [14] which, in its conventional form, does not require a mesh generation because only one single super element is used in the whole process. The difficulty

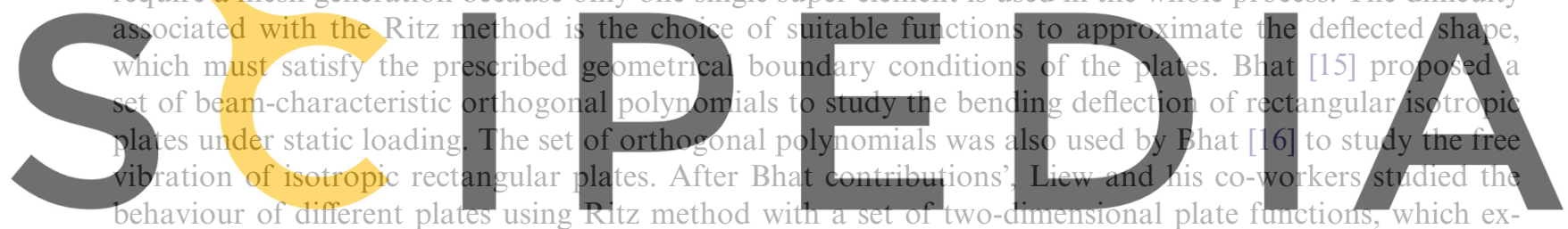

presses the entire plate domain into two implicitly related variables (see Refs. [17-28]).

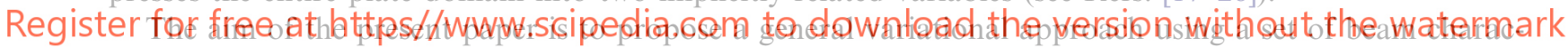

teristic orthogonal polynomials for the static and dynamical analysis of laminated composite plates having different boundary conditions. The analysis is based on the classical Kirchhoff assumptions and the use of natural coordinates in conjunction with the Ritz method to provide one single super-element which expresses the whole plate. In this way, laminates of different geometrical shapes may be represented by the mapping of a square one defined in terms of its natural coordinates. This variational approach allows to investigate the static bending behaviour and the free vibration characteristics of several composite laminated plates with any combination of boundary conditions.

To demonstrate the validity and efficiency of the proposed formulation, several numerical examples are solved and some of them are verified with results from others authors. In addition, a particular case is experimentally verified.

\section{Mathematical formulation}

\subsection{Strain, kinetic and potential energies}

Let us consider a flat, thin and composite plate with an arbitrary-shaped quadrilateral planform, as shown in Fig. 1a. The laminate is of uniform thickness $h$ and, in general, is made up of a number of layers each consisting of unidirectional fibre reinforced composite material. The fibre angle of the $k$ th layer counted from the surface $z=-h / 2$ is $\beta$ measured from the $x$ axis to the fibre orientation, with all laminate 

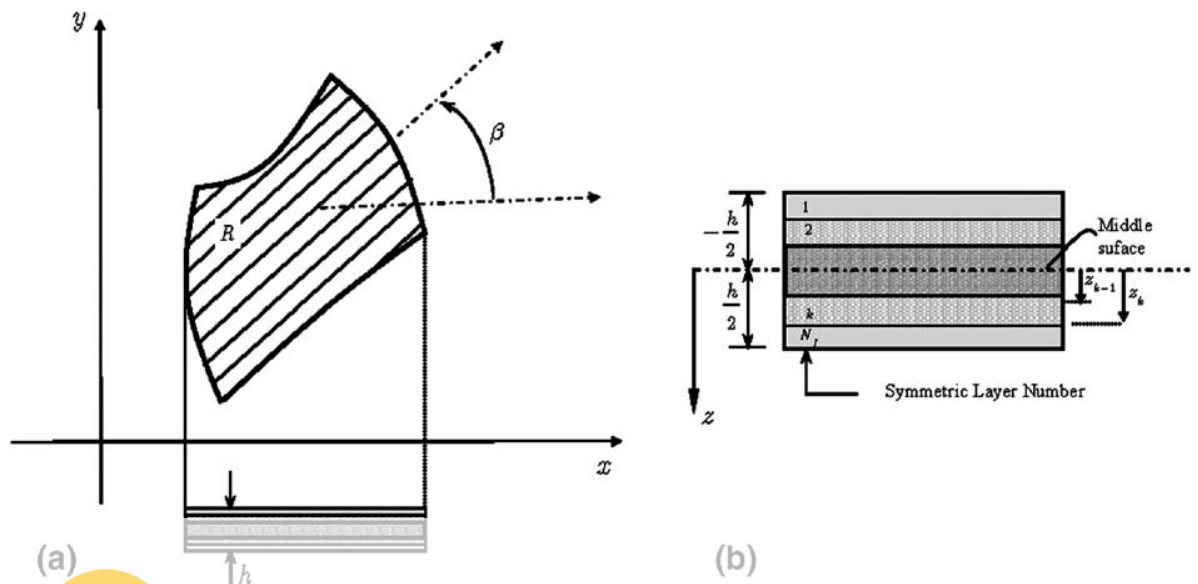

(b)

Fig. 1. (a) General description of the composite plate model. (b) Geometry of an $N$-layered symmetric laminate.

having equal thicknesses. Symmetric lamination of plies are considered, where the fibre angle of each ply is either $\beta$ or $-\beta$ such that the sequence with respect to the midplane is symmetric (Fig. 1b).

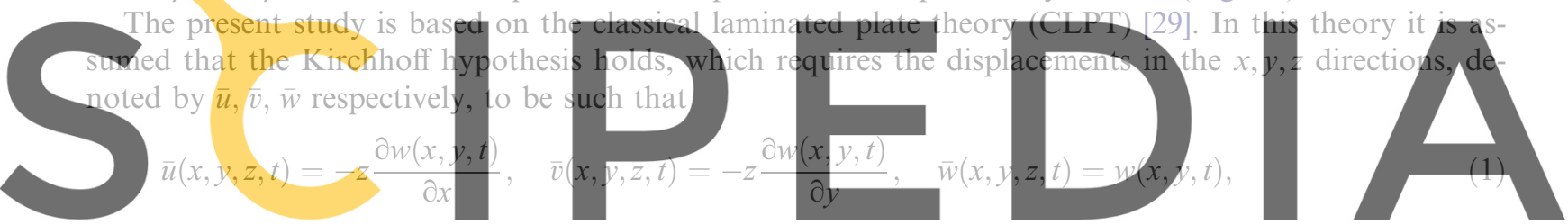

where $w(x, y, t)$ is the mid-plane plate deflection.

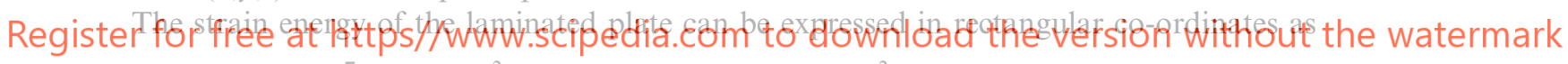

$$
\begin{aligned}
U= & \frac{1}{2} \iint_{R}\left[D_{11}\left(\frac{\partial^{2} w}{\partial x^{2}}\right)^{2}+2 D_{12} \frac{\partial^{2} w}{\partial x^{2}} \frac{\partial^{2} w}{\partial y^{2}}+D_{22}\left(\frac{\partial^{2} w}{\partial y^{2}}\right)^{2}+4 D_{16}\left(\frac{\partial^{2} w}{\partial x^{2}} \frac{\partial^{2} w}{\partial x \partial y}\right)\right. \\
& \left.+4 D_{26}\left(\frac{\partial^{2} w}{\partial y^{2}} \frac{\partial^{2} w}{\partial x \partial y}\right)+4 D_{66}\left(\frac{\partial^{2} w}{\partial x \partial y}\right)^{2}\right] \mathrm{d} x \mathrm{~d} y
\end{aligned}
$$

where the integration is carried out over the entire plate domain $R$ (see Fig. 1a) and $D_{i j}$ are the laminate stiffness coefficients and are obtained by integrating the material properties of each layer of the composite plate $[29,30]$.

The kinetic energy for free transverse vibrations of the plate is given by

$$
T=\frac{\rho h}{2} \iint_{R}\left(\frac{\partial w}{\partial t}\right)^{2} \mathrm{~d} x \mathrm{~d} y,
$$

where $\rho$ is the material density, which is considered here to be uniform through the volume of the laminate.

The deflection function is assumed periodic in time; i.e.,

$$
w(x, y, t)=W(x, y) \sin \omega t,
$$

where $\omega$ is the radian natural frequency and $W(x, y)$ is the deflection amplitude of the vibration.

The maximum strain energy $U_{\max }$ and maximum kinetic energy $T_{\max }$ in a vibratory cycle are derived by substituting Eq. (4) into Eqs. (2) and (3), respectively, whence $U_{\max }$ becomes: 


$$
\begin{aligned}
U_{\max }= & \frac{1}{2} \iint_{R}\left[D_{11}\left(\frac{\partial^{2} W}{\partial x^{2}}\right)^{2}+2 D_{12} \frac{\partial^{2} W}{\partial x^{2}} \frac{\partial^{2} W}{\partial y^{2}}+D_{22}\left(\frac{\partial^{2} W}{\partial y^{2}}\right)^{2}+4 D_{16}\left(\frac{\partial^{2} W}{\partial x^{2}} \frac{\partial^{2} W}{\partial x \partial y}\right)\right. \\
& \left.+4 D_{26}\left(\frac{\partial^{2} W}{\partial y^{2}} \frac{\partial^{2} W}{\partial x \partial y}\right)+4 D_{66}\left(\frac{\partial^{2} W}{\partial x \partial y}\right)^{2}\right] \mathrm{d} x \mathrm{~d} y,
\end{aligned}
$$

and $T_{\max }$ becomes

$$
T_{\max }=\frac{\rho h \omega^{2}}{2} \iint_{R} W^{2} \mathrm{~d} x \mathrm{~d} y .
$$

For the statical analysis of the laminate, let us consider the potential energy of a transversal load $q(x, y)$ distributed over the plate surface, which is given by

$$
V=-\iint_{R} q(x, y) W \mathrm{~d} x \mathrm{~d} y \text {. }
$$

\subsection{Transformation of coordinates}

An arbitrarily shaped quadrilateral plate in the Cartesian coordinates may be expressed simply by map-

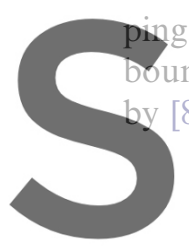

g a parent square plate

oundary equations $\xi= \pm 1$ and $\eta$ $[8,9]$ :
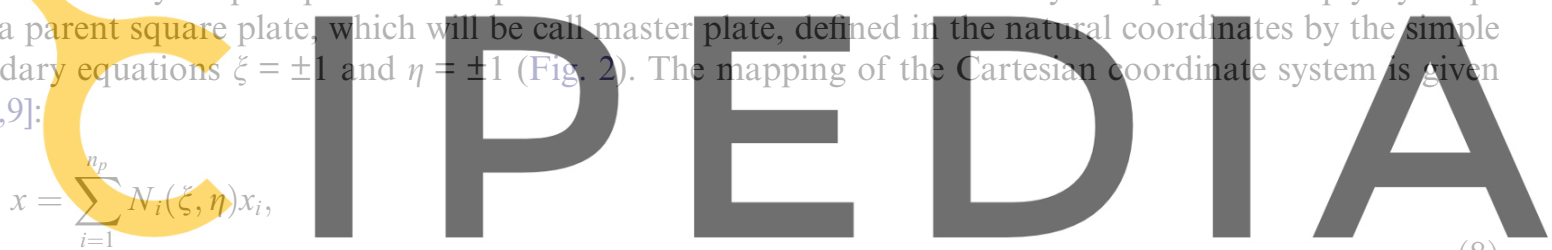

(8)

Register fop free at ifteplis//www.scipedia.com to download the version without the watermark

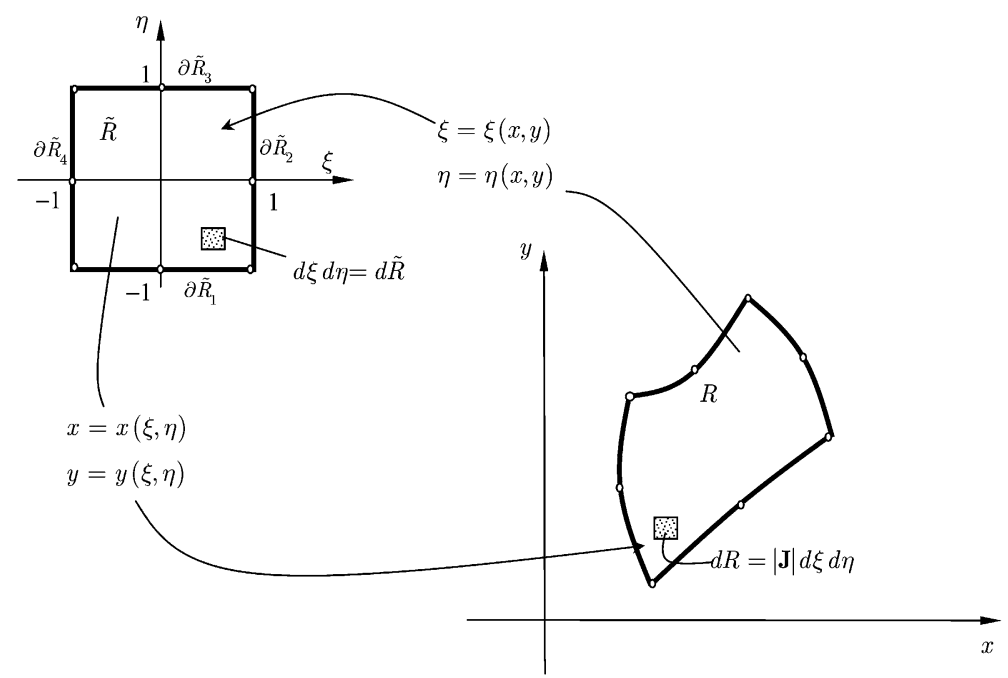

Fig. 2. Mapping of an arbitrary quadrilateral plate into natural coordinates. 
where $\left(x_{i}, y_{i}\right), i=1, \ldots, n_{p}$ are the coordinates of $n_{p}$ points on the boundary of the quadrilateral region $R$ and $N_{i}(\xi, \eta)$ are the interpolation functions of the serendipity family [8,9]. The transformation (8) maps a point $(\xi, \eta)$ in the master plate $\tilde{R}$ onto a point $(x, y)$ in the real plate domain $R$, and vice versa if the Jacobian of the transformation given by:

$$
|\mathbf{J}|=\frac{\partial x}{\partial \xi} \frac{\partial y}{\partial \eta}-\frac{\partial x}{\partial \eta} \frac{\partial y}{\partial \xi}
$$

is positive.

Applying the chain rule of differentiation it can be shown that the second derivatives of a function $W(x, y)$ are related by

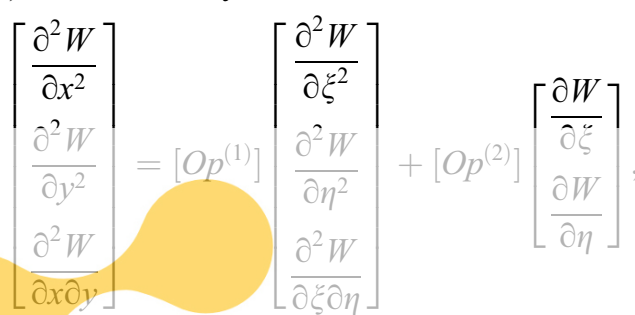

where $\left[O p^{(1)}\right]$ and $\left[O p^{(2)}\right]$ are the derivate transformation matrixes which are defined in Appendix A.

Besides, the elemental area $\mathrm{d} x \mathrm{~d} y$ in the real plate domain $R$ is transformed into $|\mathrm{J}| \mathrm{d} \xi \mathrm{d} \eta$. Consequently, the maximum kinetic energy expression given by Eq. (6) and the potential energy given by Eq. (7) reduce,
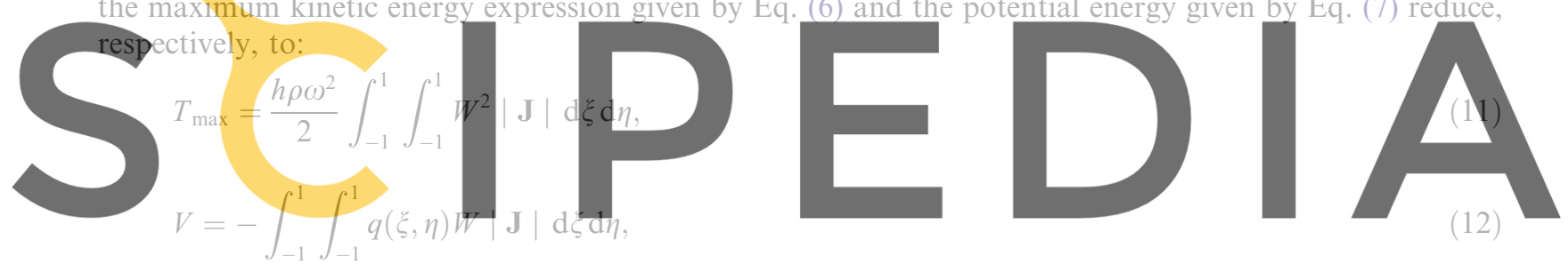

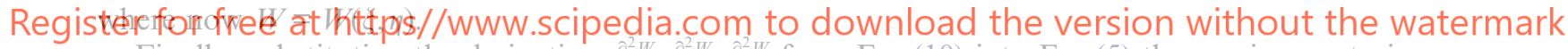

Finally, substituting the derivatives $\frac{\partial^{2} W}{\partial x^{2}}, \frac{\partial^{2} W}{\partial y^{2}}, \frac{\partial^{2} W}{\partial x \partial y}$ from Eq. (10) into Eq. (5) the maximum strain energy expression becomes

$$
\begin{aligned}
U_{\max }= & \frac{1}{2} \int_{-1}^{1} \int_{-1}^{1}\left[\left(\frac{\partial^{2} W}{\partial \xi^{2}}\right)^{2} S_{1}+\left(\frac{\partial^{2} W}{\partial \eta^{2}}\right)^{2} S_{2}+\frac{\partial^{2} W}{\partial \xi^{2}} \frac{\partial^{2} W}{\partial \eta^{2}} S_{3}+\left(\frac{\partial^{2} W}{\partial \xi \partial \eta}\right)^{2} S_{4}\right. \\
& +\frac{\partial^{2} W}{\partial \xi^{2}} \frac{\partial^{2} W}{\partial \xi \partial \eta} S_{5}+\frac{\partial^{2} W}{\partial \eta^{2}} \frac{\partial^{2} W}{\partial \xi \partial \eta} S_{6}+\frac{\partial^{2} W}{\partial \xi^{2}} \frac{\partial W}{\partial \xi} S_{7}+\frac{\partial^{2} W}{\partial \eta^{2}} \frac{\partial W}{\partial \eta} S_{8}+\frac{\partial^{2} W}{\partial \xi^{2}} \frac{\partial W}{\partial \eta} S_{9}+\frac{\partial^{2} W}{\partial \eta^{2}} \frac{\partial W}{\partial \xi} S_{10} \\
& \left.+\frac{\partial^{2} W}{\partial \xi \partial \eta} \frac{\partial W}{\partial \xi} S_{11}+\frac{\partial^{2} W}{\partial \xi \partial \eta} \frac{\partial W}{\partial \eta} S_{12}+\left(\frac{\partial W}{\partial \xi}\right)^{2} S_{13}+\left(\frac{\partial W}{\partial \eta}\right)^{2} S_{14}+\frac{\partial W}{\partial \xi} \frac{\partial W}{\partial \eta} S_{15}\right]|\mathbf{J}| \mathrm{d} \xi \mathrm{d} \eta
\end{aligned}
$$

where $S_{i}(i=1, \ldots, 15)$ are functions that depend on the problem parameters, i.e., geometry and material coefficients of the plate, and are defined in Appendix B.

The total energy functionals for free vibration and transverse bending of the plate are respectively given by:

$$
\begin{aligned}
& F_{\mathrm{d}}=U_{\max }-T_{\max }, \\
& F_{\mathrm{s}}=U+V,
\end{aligned}
$$

which are to be minimised according to the Ritz principle, as will be discussed in following sections. 


\subsection{Boundary conditions and the approximating function}

Grossi and Nallim [31,32] determined the boundary conditions which correspond to the boundary and the boundary-eingenvalue problems that describe the static and dynamic behaviour of anisotropic plates with non-smooth boundaries. In addition to these geometric and natural boundary conditions which correspond to free, clamped and simply supported edges; they determined that when a plate has a corner formed by the intersection of two free edges, unstable additional corner conditions must be considered. In the application of the Ritz method only the essential boundary conditions are required to be satisfied by the assumed functions [14]. The fact that the natural boundary conditions need not be satisfied by the chosen coordinate functions is a very important characteristic of the Ritz method, specially when dealing with problems for which these satisfaction is very difficult to achieve [33,34]. For instance, this is the case of a rectangular simply supported anisotropic plate where, the Navier analytical solution does not work because of the presence of the bending-twisting coupling: $D_{16}, D_{26}$.

The use of beam orthogonal polynomials to study anisotropic rectangular plates is very satisfactory, as has been demonstrated by Nallim and Grossi [31,35], since the convergence of the solution is rapid and practically without oscillations. This is also true in the response which requires derivates of the deflections. For this reason, in the present paper the transverse deflection of the plate is expressed in terms of the natural coordinates system by a set of beam characteristic orthogonal polynomials, $\left\{p_{i}(\xi)\right\}$ and $\left\{q_{j}(\eta)\right\}$, as
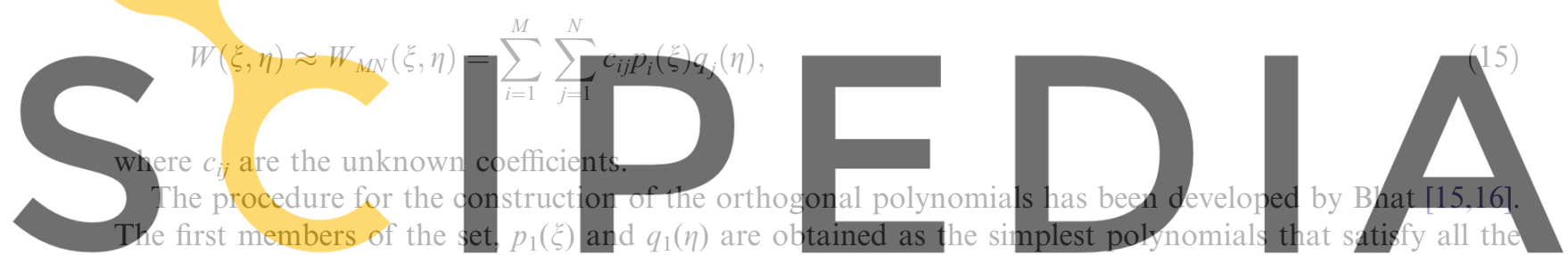

geometrical boundary conditions of the plate in their respective $\xi$ and $\eta$-directions of the natural coordinate

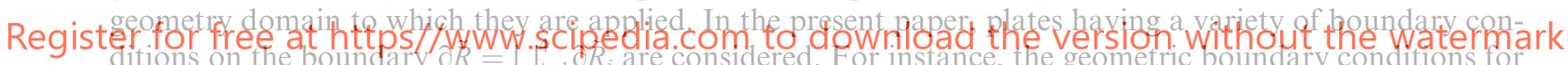
ditions on the boundary $R=U_{i=1} R_{i}$ are considered. For instance, the geometric boundary conditions for ported edge along $\partial \tilde{R}_{2}$ there is only one geometric boundary condition given by $\left.p_{1}(\xi)\right|_{\xi=1}=0$. No geometric boundary conditions exist for the free edges.

The starting polynomial of the set in the $\xi$ direction is as follows:

$$
p_{1}(\xi)=\sum_{i=0}^{I} a_{i} \xi^{i}
$$

where $I$ is equal to the total number of geometric boundary conditions on the two opposite edges. The arbitrary constants $a_{i}$, are determined by substituting Eq. (16) in the corresponding boundary conditions. The starting polynomial of the set in the $\eta$ direction, $q_{1}(\eta)$ is constructed in the same way.

The higher members of the set $\left\{p_{i}(\xi)\right\}$ are constructed by employing the Gram-Schmidt orthogonalisation procedure:

$$
p_{2}(\xi)=\left(\xi-B_{2}\right) p_{1}(\xi), \quad p_{k}(\xi)=\left(\xi-B_{k}\right) p_{k-1}(\xi)-C_{k} p_{k-2}(\xi),
$$

where

$$
B_{k}=\frac{\int_{-1}^{1} \xi\left(p_{k-1}(\xi)\right)^{2} \mathrm{~d} \xi}{\int_{-1}^{1}\left(p_{k-1}(\xi)\right)^{2} \mathrm{~d} \xi}, \quad C_{k}=\frac{\int_{-1}^{1} \xi p_{k-1}(\xi) p_{k-2}(\xi) \mathrm{d} \xi}{\int_{-1}^{1}\left(p_{k-2}(\xi)\right)^{2} \mathrm{~d} \xi} .
$$


The coefficients of the polynomials are chosen in such a way as to make the polynomials orthonormal, $\int_{-1}^{1} p_{k}^{2}(\xi)=1$. The polynomials set along the $\eta$ direction is also generated using the same procedure.

It is important to point out that working with the master element in natural coordinates allows us to use the same set of orthogonal polynomials for plates of different geometric shapes. This fact makes possible a unified treatment.

\section{Application of the Ritz method}

The Ritz method is applied to determine analytical approximate solutions for laminated plates of different shapes. For the dynamical analysis the Ritz procedure requires the minimisation of the energy functional (14a) with respect to each of the $c_{i j}$ coefficients

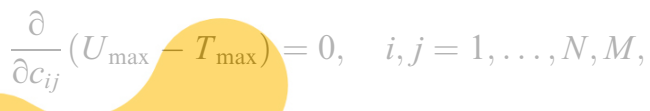

where $M, N$ are the numbers of polynomials in each natural co-ordinate.

In the same manner the statical analysis requires the minimisation of the energy functional (14b) with respect to each of the $c_{i j}$ coefficients

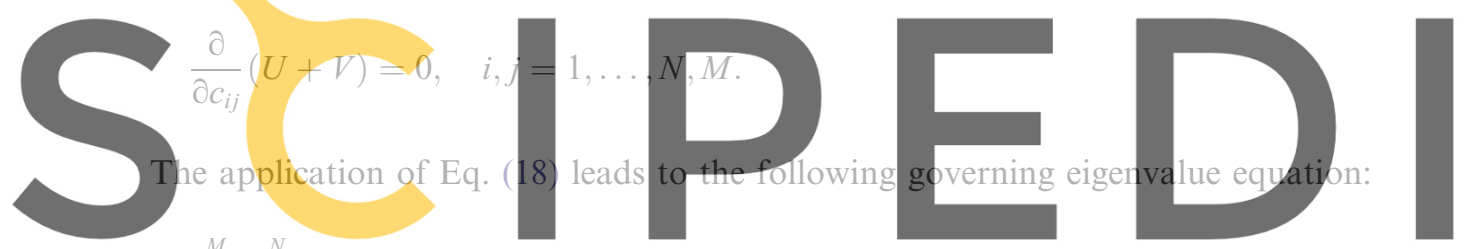

$\sum^{M} \sum^{N}\left[K_{i j k h}-\omega^{2} M_{i j k h}\right] c_{k h}=0$

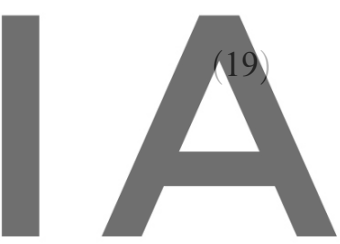

(20)

Register forlfree at https//www.scipedia.com to download the version without the watermark

On the other hand, Eq. (19) leads to the following set of $M \times N$ algebraic equations among $c_{k h}$

$$
\sum_{k=1}^{M} \sum_{h=1}^{N} K_{i j k h} c_{k h}-B_{i j}=0,
$$

where

$$
\begin{aligned}
& K_{i j k h}=\sum_{m=1}^{15} P_{i j k h, m}(\xi, \eta) \\
& B_{i j}=2 \int_{-1}^{1} \int_{-1}^{1} q(\xi, \eta) p_{i}(\xi) q_{j}(\eta)|\mathbf{J}| \mathrm{d} \xi \mathrm{d} \eta \\
& M_{i j k h}=2 \rho h \int_{-1}^{1} \int_{-1}^{1} p_{i}(\xi) p_{k}(\xi) q_{j}(\eta) q_{h}(\eta)|\mathbf{J}| \mathrm{d} \xi \mathrm{d} \eta
\end{aligned}
$$

The details of the deduction of Eqs. (20) and (21) from Eqs. (18) and (19) together with the analytical expressions of the terms $P$ 's are given in Appendix C.

Equation (20) yields an eigenvalue determinant, whose zeros give the natural frequencies of the plate. Back substitution yields the coefficient vectors; and finally substitution of these coefficient vectors into Eq. (15) gives the corresponding mode shapes of the plate. 


\section{Verification and numerical applications}

\subsection{Generalities}

A computer code, based on the variational algorithm developed in this paper, was implemented and used for the analysis of plates having different shapes, material properties and boundary conditions. The presented results correspond to the dynamical and statical analyses of the above mentioned plates. For the dynamical analysis, natural frequencies parameter and modal shapes were computed. While, for the statical analysis, deflections and bending moments were calculated under uniformly distributed loads. Although, in the present study, only plates under uniformly distributed loads are presented, the developed algorithm can handle many others applied loads.

In order to establish the accuracy and applicability of the approach described, numerical results were computed for a number of plate problems for which comparison values were available in the literature. Additionally, a great number of problems were solved and since the number of cases was extremely large, results were presented for only a few cases. Calculations have been performed taking plates with different geometrical shapes, material properties, angles of fibre orientation and stacking sequences.

Let us introduce the terminology to be used throughout the remainder of the paper for describing the boundary conditions of the plates considered. The designation $\mathrm{C}-\mathrm{S}-\mathrm{F}-\mathrm{S}$, for example, identifies a plate with edges (1) clamped, (2) simply supported, (3) free and (4) simply supported (see Fig. 3). The reference flexural rigidity is $D_{\beta}=E_{\mathrm{L}} h^{3} / 12\left(1-v_{\mathrm{LT}} v_{\mathrm{TL}}\right)$, the subscripts $\mathrm{L}$ and $\mathrm{T}$ represent the directions parallel with
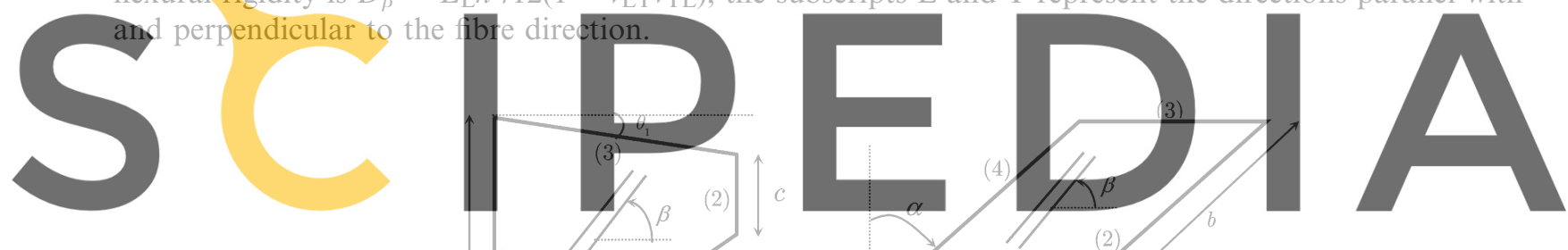

Register for free at https//Www.seipedia.com to download(the version without the watermark

(a)

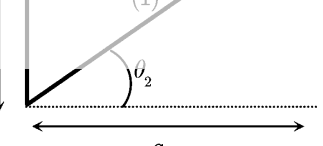

$a$

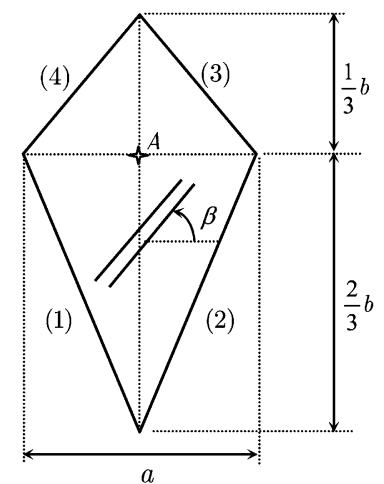

(c)

)
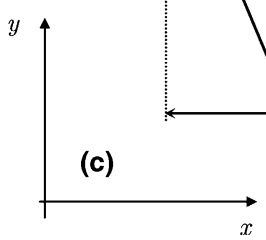

(b)

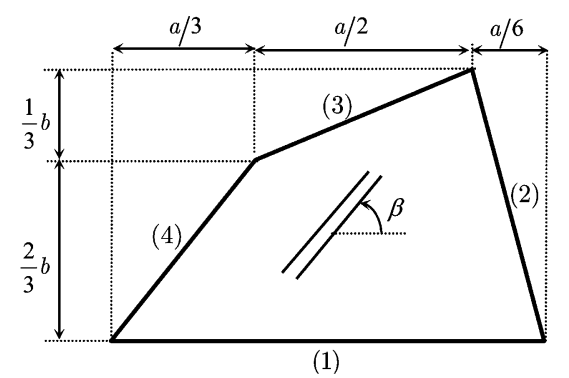

(d)

Fig. 3. Laminates of various shapes. 
The main purposes of these exercises are twofold. One is to demonstrate the accuracy and efficiency of the proposed method, and the other is to produce some results which may be regarded as benchmark solutions for other academic research workers and design engineers.

\subsection{Convergence and comparison of eigenvalues}

Results of a convergence study of eigenvalues $\omega a^{2} \sqrt{\rho h / D_{\beta}}$ are presented in Table 1. Four-ply E-glass/epoxi laminates $\left(E_{\mathrm{L}}=60.7 \mathrm{GPa}, E_{\mathrm{T}}=24.8 \mathrm{GPa}, G_{\mathrm{LT}}=12 \mathrm{GPa}, v_{\mathrm{LT}}=0.23\right)$, with stacking sequence $(\beta,-\beta,-\beta, \beta)$ are considered for $\beta=30^{\circ}$ and $60^{\circ}$. The rate of convergence of eigenvalues is shown for $\mathrm{F}-\mathrm{S}-\mathrm{F}-\mathrm{S}$ trapezoidal, skew and rhomboidal laminates. It is well known that the Ritz method gives upper bounds eigenvalues. The convergence of the mentioned eigenvalues is studied by gradually increasing the number of polynomials used in each natural co-ordinate. It can be seen that $M, N=12$, is sufficient to reach stable convergence. Moreover, $M, N=10$ produces no drastic change in the solutions compared with $M, N=12$. Therefore, it was decided to use $M, N=10$ to generate the results with sufficient accuracy from an engineering viewpoint.

The accuracy and reliability of the eigenvalues obtained with the presented approach are demonstrated in the following three cases. The comparison presented in Table 2, authenticates the validity of the present method for symmetrically laminated trapezoidal plates with $\theta_{1}=\theta_{2}$ and various chord ratios $c / b$ (see Fig. 3a). The first eight non-dimensional frequencies $\omega a b \sqrt{\rho h / D_{\beta}}$ for four-ply symmetric laminated plates with stacking sequence $(-\beta, \beta, \beta,-\beta)$ aspect ratio $a / b=2$ and subject to two different boundary conditions are tabulated in the mentioned Table. The material properties of each lamina are characterised by $E_{\mathrm{L}} / E_{\mathrm{T}}=$

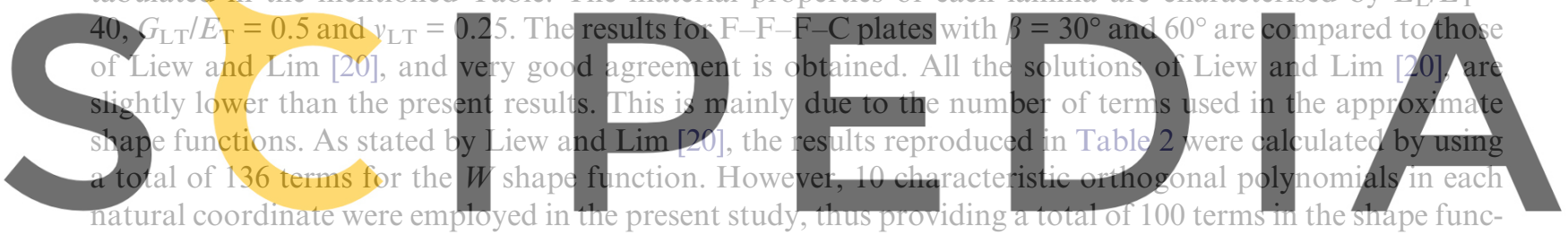

tion. Other frequencies for S-C-F-S and C-S-S-F four-ply, symmetric laminates are also included in Table 2.

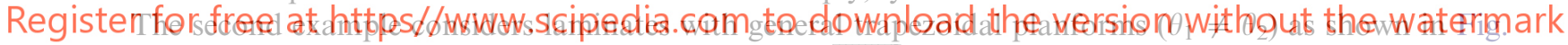

3a. The first eight non-dimensional frequencies $\omega a^{2} / h \sqrt{\rho / E_{\mathrm{L}}}$ for four-ply symmetrically laminated E-glass/ epoxi plates with stacking sequence $(-\beta, \beta, \beta,-\beta)$, aspect ratio $a / b=2$ and subject to two different boundary conditions are tabulated in Table 3 . The results for full simply supported and cantilever plates are compared to those of Lim et al. [26], and very good agreement is obtained.

Finally, the third example verifies the accuracy of the eigenvalues for thin skew fibre reinforced laminates with five symmetric angle-ply layers and stacking sequence $(45,-45,45,-45,45)$. The geometry of the skew plate is defined by means of $a, b$ and $\alpha$ as shown in Fig. 3b. The material properties of each lamina are $E_{\mathrm{L}} / E_{\mathrm{T}}=40, G_{\mathrm{LT}} / E_{\mathrm{T}}=0.6, v_{\mathrm{LT}}=0.25$ and three skew angles, i.e., $\alpha=0^{\circ}, 30^{\circ}$ and $45^{\circ}$, are used for comparison in this case. The first eight non-dimensional frequencies $\omega\left(b^{2} / h \pi^{2}\right) \sqrt{\rho / E_{\mathrm{T}}}$ obtained with the present approach, for two kinds of boundary conditions, i.e., fully simply supported (S-S-S-S) and fully clamped (C-C-C-C), are compared with the solutions of Wang [36] in Table 4. Excellent agreement is achieved between both solutions. Additional results for $\mathrm{C}-\mathrm{F}-\mathrm{F}-\mathrm{S}$ and $\mathrm{S}-\mathrm{S}-\mathrm{C}-\mathrm{F}$ skew laminates, are also included in the mentioned table.

\subsection{Comparison of nodal patterns and modal shapes}

In this section a comparison between experimental and numerical results obtained with the proposed formulation are shown. The analysed test plate has a general trapezoidal planform, it is made of an isotropic material and it is clamped on edge (4) and the other edges are free. The geometrical and material properties of the plate are specified in Table 5. The experimental results have been obtained using electronic speckle pattern interferometry (ESPI) by the Optical Laser Group (National University of Salta), the details of this 
Table 1

Convergence of frequency parameters $\omega a^{2} \sqrt{\rho h / D_{\beta}}$ for symmetrically laminated E-glass-epoxi plates with stacking sequence $(\beta,-\beta,-\beta, \beta)$

\begin{tabular}{lllllllll}
\hline$\beta$ & $M \times N$ & \multicolumn{3}{l}{ Mode sequence number } & & & \\
\cline { 2 - 8 } & 2 & 3 & 4 & 5 & 6 & 7 & 8
\end{tabular}

Trapezoidal plate, al $b=2, c / b=0.25, \theta_{1}=\theta_{2}$

$30^{\circ} \quad 6 \times 6 \quad 2.0232 \quad 7.4693$

$\begin{array}{lll}6 \times 6 & 2.0232 & 7.4693 \\ 7 \times 7 & 2.0232 & 7.4692\end{array}$

$\begin{array}{lll}7 \times 7 & 2.0232 & 7.4692 \\ 8 \times 8 & 2.0232 & 7.4691\end{array}$

$9 \times 9 \quad 2.0231 \quad 7.4691$

$10 \times 10 \quad 2.0231 \quad 7.4691$

$11 \times 11 \quad 2.0231 \quad 7.4691$

$12 \times 12 \quad 2.0231$

$10 \times 10$

$\begin{array}{rr}10 \times 10 & 1.5956 \\ 11 \times 11 & 1.5956\end{array}$

$12 \times 12 \quad 1.5956$
7.4690

6.4867

6.4773

6.4771

6.4770

6.4770
6.4770

6.4770

6.4770

\begin{abstract}
9.2771
9.2763

9.2757

9.2755

9.2753

9.2752

9.2751
\end{abstract}

8.4909

8.4802

8.4797

8.4791

8.4789

8.4787

8.4786

8.4784
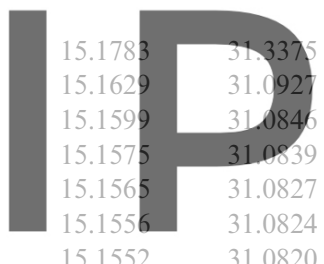

17.6020
17.4936
17.4897
17.4885
17.4883
17.4884
17.4884
15.0218
14.8122
14.6767
14.6715
14.6698
14.6697
14.6696
14.6696

21.2079
21.0931
21.0844
21.0826
21.0820
21.0816
21.0814

19.2174

19.1379

19.1012

19.0990

19.0980

19.0976

19.0972

19.0970

31.4849
31.0497
30.6832
30.6643
30.6547
30.6543
30.6542

33.1934

27.3909

26.8725

26.1922

26.1592

26.1405

26.1397

26.1395
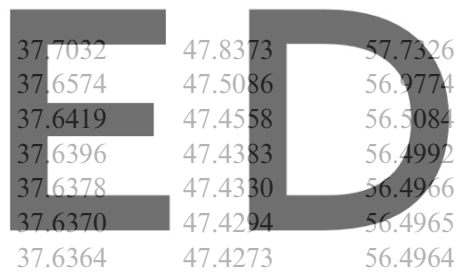

$\begin{array}{ll}35.4808 & 37.6965 \\ 34.8383 & 37.2965 \\ 34.6169 & 36.7673 \\ 34.5845 & 36.7423 \\ 34.5770 & 36.7288 \\ 34.5760 & 36.7275 \\ 34.5758 & 36.7268 \\ 40.1868 & 43.7078 \\ 32.2560 & 40.1359 \\ 32.1018 & 39.5945 \\ 31.8788 & 39.5786 \\ 31.8675 & 39.4884 \\ 31.8618 & 39.4838 \\ 31.8607 & 39.4746 \\ 31.8599 & 39.4744\end{array}$

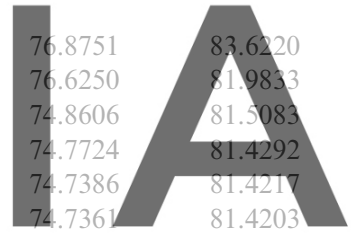

74.7343

81.4192

Register for free at httigs 3 / w ww

$\begin{array}{rr}7 \times 7 & 8.4004 \\ 8 \times 8 & 8.3990 \\ 9 \times 9 & 8.3982 \\ 10 \times 10 & 8.3978 \\ 11 \times 11 & 8.3975 \\ 12 \times 12 & 8.3973\end{array}$
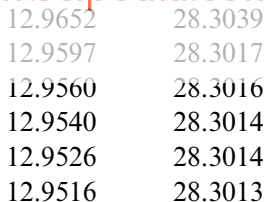

Rhomboidal plate, $a / b=1$

$30^{\circ}$

$\begin{array}{rr}6 \times 6 & 12.6745 \\ 7 \times 7 & 12.6744 \\ 8 \times 8 & 12.6744 \\ 9 \times 9 & 12.6744 \\ 10 \times 10 & 12.6744 \\ 11 \times 11 & 12.6744 \\ 12 \times 12 & 12.6744\end{array}$

26.5317

26.5273

26.5263

26.5255

26.5252

26.5250

26.5249

$60^{\circ}$

$\begin{array}{rrr}6 \times 6 & 12.8008 & 26.368 \\ 7 \times 7 & 12.8006 & 26.343 \\ 8 \times 8 & 12.8006 & 26.334 \\ 9 \times 9 & 12.8005 & 26.327 \\ 10 \times 10 & 12.8005 & 26.324 \\ 11 \times 11 & 12.8005 & 26.3214 \\ 12 \times 12 & 12.8005 & 26.3197\end{array}$

47.7675
47.7402
47.7384
47.7383
47.7382
47.7382
47.7381
48.9445
48.9333
48.9317
48.9315
48.9315
48.9314
48.9314

63.2555

62.4949

62.4838

62.4814

62.4802

62.4796

62.4791

60.7346

60.1017

60.0845

60.0778

60.0724

60.0697

60.0673

\subsection{6538}

45.6333

45.6230

45.6166

45.6122
50.5577
50.4242

50.4196

50.4185

50.4183

50.4180

\subsection{4}

71.8148

71.8077

71.8052

71.8034
79.6427

.0421

77.9019

77.8799

77.8789

77.8787

\begin{tabular}{llll}
75.6158 & 103.2656 & 117.0172 & 136.4726 \\
75.5491 & 101.8325 & 115.2071 & 136.2466 \\
75.5313 & 101.7452 & 114.9393 & 132.4501 \\
75.5295 & 101.7267 & 114.9204 & 132.4088 \\
75.5293 & 101.7250 & 114.9138 & 132.3737 \\
75.5291 & 101.7246 & 114.9128 & 132.3725 \\
75.5290 & 101.7244 & 114.9122 & 132.3717 \\
76.6698 & 107.9375 & 112.0938 & 134.5834 \\
76.5243 & 106.1860 & 110.7694 & 134.5029 \\
76.5017 & 106.1573 & 110.5055 & 130.5065 \\
76.4913 & 106.1316 & 110.4856 & 130.4779 \\
76.4862 & 106.1314 & 110.4712 & 130.4293 \\
76.4821 & 106.1311 & 110.4642 & 130.4242 \\
76.4796 & 106.1311 & 110.4581 & 130.4204 \\
\hline
\end{tabular}


Table 2

Frequency parameters $\omega a b \sqrt{\frac{\rho h}{D_{\beta}}}$ for trapezoidal composite laminates with four symmetric angle-ply layers $(-\beta, \beta, \beta,-\beta)$ and with $a / b=2$

\begin{tabular}{|c|c|c|c|c|c|c|c|c|c|c|}
\hline \multirow[t]{2}{*}{$c / b$} & \multirow[t]{2}{*}{$\beta$} & & \multicolumn{8}{|c|}{ Mode sequence number } \\
\hline & & & 1 & 2 & 3 & 4 & 5 & 6 & 7 & 8 \\
\hline \multicolumn{11}{|c|}{$\overline{F-F-F-C}$} \\
\hline \multirow[t]{4}{*}{0.25} & $30^{\circ}$ & Present & 1.4292 & 6.5088 & 11.153 & 17.447 & 25.572 & 34.185 & 41.231 & 46.183 \\
\hline & & Liew and Lim [20] & 1.4285 & 6.5068 & 11.150 & 17.443 & 25.567 & 34.178 & 41.228 & 46.161 \\
\hline & $60^{\circ}$ & Present & 0.5242 & 2.5003 & 6.6330 & 9.1509 & 13.133 & 21.005 & 22.426 & 32.954 \\
\hline & & Liew and Lim [20] & 0.5229 & 2.4973 & 6.6286 & 9.1407 & 13.127 & 20.987 & 22.399 & 32.763 \\
\hline \multirow[t]{4}{*}{0.50} & $30^{\circ}$ & Present & 1.2114 & 5.9881 & 8.2722 & 16.103 & 21.037 & 29.684 & 33.877 & 38.347 \\
\hline & & Liew and Lim [20] & 1.2109 & 5.9875 & 8.2713 & 16.102 & 21.035 & 29.683 & 33.873 & 38.344 \\
\hline & $60^{\circ}$ & Present & 0.44363 & 2.3685 & 6.5152 & 6.6008 & 13.226 & 16.454 & 22.346 & 27.560 \\
\hline & & Liew and Lim [20] & 0.44283 & 2.3669 & 6.5092 & 6.5984 & 13.222 & 16.441 & 22.324 & 27.539 \\
\hline \multirow[t]{4}{*}{0.75} & $30^{\circ}$ & Present & 1.0890 & 5.2800 & 6.7605 & 14.454 & 17.553 & 25.360 & 26.235 & 33.702 \\
\hline & & Liew and Lim [20] & 1.0891 & 5.2799 & 6.7618 & 14.454 & 17.555 & 25.359 & 26.233 & 33.701 \\
\hline & $60^{\circ}$ & Present & 0.39887 & 2.2993 & 4.8515 & 6.5949 & 13.083 & 13.817 & 22.078 & 23.712 \\
\hline & & Liew and Lim [20] & 0.39842 & 2.2987 & 4.8475 & 6.5939 & 13.077 & 13.811 & 22.062 & 23.699 \\
\hline \multicolumn{11}{|c|}{$S-C-F-S$} \\
\hline \multirow[t]{2}{*}{0.25} & $30^{\circ}$ & Present & 8.8250 & 20.7205 & 31.2324 & 39.4444 & 50.9814 & 63.5851 & 7.0219 & 79.2545 \\
\hline & $60^{\circ}$ & Present & 6.4225 & 14.0281 & 23.3516 & 34.5737 & 46.2103 & 49.5273 & 63.4942 & 69.0133 \\
\hline \multirow[t]{2}{*}{0.50} & $30^{\circ}$ & Present & 8.1296 & 18.8568 & 26.3554 & 34.8923 & 44.0993 & 55.3712 & 59.5676 & 68.5765 \\
\hline & $60^{\circ}$ & Present & 5.7021 & 12.3459 & 20.6233 & 30.7605 & 40.1498 & 43.5531 & 54.1623 & 58.9306 \\
\hline \multirow[t]{2}{*}{0.75} & $30^{\circ}$ & Present & 7.6040 & 17.1370 & 23.0643 & 31.2288 & 40.1085 & 49.0695 & 49.9709 & 61.5169 \\
\hline & $60^{\circ}$ & Present & 5.1417 & 11.2012 & 18.9364 & 28.5030 & 33.4761 & 39.7395 & 43.6576 & 52.2321 \\
\hline \multicolumn{11}{|c|}{$C-S-S-F$} \\
\hline \multirow[t]{2}{*}{0.25} & $30^{\circ}$ & Present & 13.6706 & 30.8146 & 40.3287 & 51.0371 & 66.1227 & 75.9397 & 86.2925 & 95.6579 \\
\hline & $60^{\circ}$ & Present & 29.2473 & 47.6310 & 64.1050 & 81.0669 & 90.0585 & 108.6065 & 124.7442 & 139.090 \\
\hline \multirow[t]{2}{*}{0.50} & $30^{\circ}$ & Present & 12.5154 & 24.8775 & 35.4636 & 40.2675 & 54.7443 & 61.6343 & 73.0903 & 78.5758 \\
\hline & $60^{\circ}$ & Present & 27.6866 & 40.7856 & 51.9092 & 63.1236 & 74.7997 & 84.3329 & 93.3935 & 110.552 \\
\hline \multirow[t]{2}{*}{0.75} & $30^{\circ}$ & Present & 11.2647 & 20.0165 & 30.9280 & 34.6424 & 47.5583 & 50.1971 & 62.8488 & 69.7796 \\
\hline & $60^{\circ}$ & Present & 25.9214 & 33.5302 & 40.0809 & 48.1569 & 58.5164 & 71.1055 & 80.7580 & 94.9314 \\
\hline
\end{tabular}

experimental technique can be found in Ref. [37]. In Fig. 4 experimentally obtained modal shapes are compared to analytical predictions, obtained with the proposed method for eight of the natural frequencies of free vibration. It can be seen a remarkable agreement between the calculated modal shapes and those obtained by means the ESPI.

\subsection{Rhomboidal plates}

In this section, results are presented of the developed approach applied to study the statical and dynamical behaviour of rhomboidal laminates as shown in Fig. 3c. The planform geometry of the rhomboidal plate is defined by means of the aspect ratio b/a. Four-ply E-glass/epoxi laminates $\left(E_{\mathrm{L}}=60.7 \mathrm{GPa}\right.$, $\left.E_{\mathrm{T}}=24.8 \mathrm{GPa}, G_{\mathrm{LT}}=12 \mathrm{GPa}, v_{\mathrm{LT}}=0.23\right)$, with stacking sequence $(\beta,-\beta,-\beta, \beta)$ are considered. As shown in Table 6 three different combinations of boundary conditions are taking into account. In each category of 
Table 3

Frequency parameters $\frac{\omega a^{2}}{h} \sqrt{\frac{\rho}{E_{\mathrm{L}}}}$, for general trapezoidal composite laminates with four symmetric angle-ply layers $(-\beta, \beta, \beta,-\beta)$ and with $a / b=2$

\begin{tabular}{|c|c|c|c|c|c|c|c|c|c|c|c|}
\hline \multirow[t]{2}{*}{$\frac{c}{b}$} & \multirow[t]{2}{*}{$\theta_{1}$} & \multirow[t]{2}{*}{$\beta$} & & \multicolumn{8}{|c|}{ Mode sequence number } \\
\hline & & & & 1 & 2 & 3 & 4 & 5 & 6 & 7 & 8 \\
\hline \multicolumn{12}{|c|}{$S-S-S-S$} \\
\hline \multirow[t]{16}{*}{0.25} & $0^{\circ}$ & $0^{\circ}$ & Present & 19.0701 & 33.8324 & 50.7168 & 55.9894 & 71.9130 & 82.0421 & 97.4327 & 106.214 \\
\hline & & & Lim et al. [26] & 19.070 & 33.832 & 50.718 & 55.988 & 71.852 & 82.017 & 96.895 & 105.98 \\
\hline & & $30^{\circ}$ & Present & 21.1688 & 35.9472 & 52.3902 & 62.9348 & 72.8788 & 88.3813 & 97.8691 & 114.105 \\
\hline & & & Lim et al. [26] & 21.168 & 35.947 & 52.387 & 62.928 & 72.741 & 88.237 & 97.295 & 112.86 \\
\hline & & $60^{\circ}$ & Present & 23.4112 & 37.3871 & 52.9366 & 69.3333 & 74.0400 & 90.5069 & 99.9535 & 119.4007 \\
\hline & & & Lim et al. [26] & 23.411 & 37.386 & 52.916 & 69.233 & 73.926 & 89.683 & 99.628 & 112.27 \\
\hline & & $90^{\circ}$ & Present & 23.2906 & 36.8643 & 52.0483 & 68.9188 & 73.1376 & 89.0716 & 99.0967 & 118.566 \\
\hline & & & Lim et al. [26] & 23.290 & 36.863 & 52.020 & 68.774 & 73.099 & 87.969 & 98.949 & 109.26 \\
\hline & $5^{\circ}$ & $0^{\circ}$ & Present & 18.6992 & 33.4684 & 50.8896 & 54.3244 & 72.2477 & 80.6193 & 98.0837 & 105.4503 \\
\hline & & & Lim et al. [26] & 18.699 & 33.468 & 50.890 & 54.323 & 72.193 & 80.607 & 97.564 & 105.36 \\
\hline & & $30^{\circ}$ & Present & 20.4967 & 35.4311 & 52.3098 & 60.5054 & 72.8539 & 87.3175 & 97.3830 & 113.2849 \\
\hline & & & Lim et al. [26] & 20.497 & 35.431 & 52.308 & 60.503 & 72.739 & 87.263 & 96.896 & 112.74 \\
\hline & & $60^{\circ}$ & Present & 22.8287 & 36.9593 & 52.7782 & 68.8655 & 72.1939 & 90.9500 & 97.8008 & 119.9634 \\
\hline & & & Lim et al. [26] & 22.829 & 36.959 & 52.763 & 68.822 & 72.067 & 90.295 & 97.637 & 113.22 \\
\hline & & $90^{\circ}$ & Present & 23.0681 & 36.6096 & 51.8279 & 68.9544 & 72.1472 & 88.9842 & 98.1283 & 118.5196 \\
\hline & & & Lim et al. [26] & 23.068 & 36.609 & 51.802 & 68.803 & 72.132 & 87.975 & 98.074 & 109.26 \\
\hline \multicolumn{12}{|c|}{$F-F-F-C$} \\
\hline \multirow[t]{16}{*}{0.5} & $0^{\circ}$ & $0^{\circ}$ & Present & 1.2221 & 5.6951 & 6.8472 & 15.3477 & 18.5433 & 28.3769 & 33.8152 & 35.6139 \\
\hline & & & Lim et al. [26] & 1.2221 & 5.6950 & 6.8471 & 15.348 & 18.543 & 28.376 & 33.815 & 35.613 \\
\hline & & $30^{\circ}$ & Present & 1.0061 & 5.2731 & 6.7338 & 13.9645 & 17.8660 & 26.1989 & 32.3001 & 37.4719 \\
\hline & & & Lim et al. [26] & 1.0061 & 5.2730 & 6.7339 & 13.964 & 17.866 & 26.197 & 32.300 & 37.469 \\
\hline & & $60^{\circ}$ & Present & 0.82147 & 4.44336 & 6.06730 & 11.9046 & 15.7535 & 22.9109 & 27.5990 & 37.1082 \\
\hline & & & Lim et al. [26] & 0.82145 & 4.4432 & 6.0671 & 11.904 & 15.753 & 22.909 & 27.598 & 37.097 \\
\hline & & $90^{\circ}$ & Present & 0.78989 & 4.2837 & 5.4574 & 11.4872 & 14.2802 & 22.1285 & 25.2617 & 35.8900 \\
\hline & & & Lim et al. [26] & 0.78987 & 4.2836 & 5.4573 & 11.487 & 14.280 & 22.128 & 25.261 & 35.883 \\
\hline & $5^{\circ}$ & $0^{\circ}$ & Present & 1.2441 & 5.8082 & 6.7956 & 15.8474 & 18.2620 & 29.4464 & 33.1838 & 35.3629 \\
\hline & & & Lim et al. [26] & 1.2441 & 5.8081 & 6.7955 & 15.847 & 18.262 & 29.446 & 33.183 & 35.362 \\
\hline & & $30^{\circ}$ & Present & 1.0396 & 5.4977 & 6.6585 & 14.6412 & 17.7271 & 27.5625 & 32.3319 & 36.3853 \\
\hline & & & Lim et al. [26] & 1.0395 & 5.4976 & 6.6585 & 14.641 & 17.727 & 27.561 & 32.332 & 36.384 \\
\hline & & $60^{\circ}$ & Present & 0.83427 & 4.5300 & 6.1437 & 12.1786 & 15.9430 & 23.5677 & 27.8769 & 38.4930 \\
\hline & & & Lim et al. [26] & 0.83424 & 4.5299 & 6.1435 & 12.178 & 15.942 & 23.566 & 27.876 & 38.483 \\
\hline & & $90^{\circ}$ & Present & 0.79708 & 4.3421 & 5.4137 & 11.6678 & 14.1548 & 22.5941 & 24.9532 & 37.0395 \\
\hline & & & Lim et al. [26] & 0.79706 & 4.3420 & 5.4136 & 11.668 & 14.155 & 22.593 & 24.953 & 37.033 \\
\hline
\end{tabular}

boundary conditions two aspect ratios, i.e., $b / a=1$ and 2 are considered, and the angle of fibre orientation ranges from $\beta=0^{\circ}$ to $90^{\circ}$. In this table, $\beta=0^{\circ}$ and $90^{\circ}$ mean cross-ply laminates with stacking sequences $\left(0^{\circ}, 90^{\circ}, 90^{\circ}, 0^{\circ}\right)$ or $\left(90^{\circ}, 0^{\circ}, 0^{\circ}, 90^{\circ}\right)$ respectively.

For the statical analysis, deflections and bending moments in an specific point of the rhomboidal plate (point marked by $A$ in Fig. 3c), under uniform distributed load $q$ are calculated. For the dynamical analysis, the first eight natural frequencies of free vibrations are determined.

As can be observed in Table 6 , for S-S-S-S laminates with $b / a=1$ the lowest fundamental frequency occurs for $\beta=45^{\circ}$. On the other hand, the maximum values are obtained for the cross-ply configurations. For the same aspect ratio $b / a=1$, but $\mathrm{C}-\mathrm{C}-\mathrm{C}-\mathrm{C}$ boundary condition, the dynamical behaviour of the laminate with regards to the angle of fibre orientation, is quite different. In this case, the fundamental frequency 
Table 4

Frequency parameters $\frac{\omega b^{2}}{h \pi^{2}} \sqrt{\frac{\rho}{E_{\mathrm{T}}}}$, for skew composite laminates with five symmetric angle-ply layers $\left(45^{\circ},-45,45,-45,45\right)$ and with $a=b$

\begin{tabular}{|c|c|c|c|c|c|c|c|c|c|}
\hline \multirow[t]{2}{*}{$\alpha$} & & \multicolumn{8}{|c|}{ Mode sequence number } \\
\hline & & 1 & 2 & 3 & 4 & 5 & 6 & 7 & 8 \\
\hline \multicolumn{10}{|c|}{$S-S-S-S$} \\
\hline \multirow[t]{2}{*}{$0^{\circ}$} & Present & 2.4339 & 4.9865 & 6.1820 & 8.4869 & 10.2535 & 11.6467 & 12.8259 & 15.2168 \\
\hline & Wang [36] & 2.4339 & 4.9865 & 6.1818 & 8.4870 & 10.2536 & 11.6464 & 12.8260 & 15.2173 \\
\hline \multirow[t]{2}{*}{$30^{\circ}$} & Present & 2.6099 & 5.6869 & 6.8246 & 9.4721 & 11.8822 & 13.2191 & 14.2739 & 17.3240 \\
\hline & Wang [36] & 2.6119 & 5.6902 & 6.8316 & 9.4773 & 11.8900 & 13.2355 & 14.2809 & 17.3382 \\
\hline \multirow[t]{2}{*}{$45^{\circ}$} & Present & 3.3192 & 6.9005 & 9.6936 & 10.7209 & 15.5313 & 16.1444 & 19.3509 & 21.2960 \\
\hline & Wang [36] & 3.3182 & 6.9002 & 9.6908 & 10.7206 & 15.5318 & 16.1447 & 19.3481 & 21.3005 \\
\hline \multicolumn{10}{|c|}{$C-C-C-C$} \\
\hline \multirow[t]{2}{*}{$0^{\circ}$} & Present & 3.9009 & 7.1463 & 8.4583 & 11.2109 & 13.3212 & 14.7414 & 16.1260 & 18.8114 \\
\hline & Wang [36] & 3.9009 & 7.1464 & 8.4585 & 11.2112 & 13.3216 & 14.7425 & 16.1271 & 18.8145 \\
\hline \multirow[t]{2}{*}{$30^{\circ}$} & Present & 4.5389 & 8.3765 & 9.8697 & 12.8450 & 15.6794 & 17.4656 & 18.3284 & 21.9142 \\
\hline & Wang [36] & 4.5431 & 8.3819 & 9.8810 & 12.8533 & 15.6906 & 17.4889 & 18.3396 & 21.9364 \\
\hline \multirow[t]{2}{*}{$45^{\circ}$} & Present & 6.3046 & 10.8189 & 14.4932 & 15.4684 & 21.0550 & 22.0641 & 25.8787 & 27.6348 \\
\hline & Wang [36] & 6.3048 & 10.8193 & 14.4949 & 15.4692 & 21.0620 & 22.0759 & 25.8849 & 27.6869 \\
\hline \multicolumn{10}{|c|}{$C-F-F-S$} \\
\hline $0^{\circ}$ & Present & 0.61806 & 1.7646 & 2.8216 & 4.2823 & 5.2460 & 6.8291 & 7.6222 & 9.3103 \\
\hline $30^{\circ}$ & Present & 0.71969 & 1.9514 & 3.1401 & 4.5279 & 6.3471 & 7.6705 & 8.4890 & 10.6722 \\
\hline $45^{\circ}$ & Present & 0.67251 & 2.0793 & 4.0722 & 4.4189 & 7.5341 & 8.7412 & 11.1661 & 11.7505 \\
\hline \multicolumn{10}{|c|}{$S-S-C-F$} \\
\hline $0^{\circ}$ & Present & 1.7740 & 3.6275 & 5.1019 & 6.5743 & 8.0841 & 10.0992 & 10.6657 & 12.4583 \\
\hline $30^{\circ}$ & Present & 2.1794 & 4.0524 & 6.5728 & 7.3611 & 9.2862 & 11.6196 & 13.4776 & 14.4630 \\
\hline $45^{\circ}$ & Present & 2.8343 & 4.8471 & 8.4746 & 9.1855 & 12.3188 & 12.9092 & 17.7200 & 18.4634 \\
\hline
\end{tabular}

Table 5

Mechanical and geometrical properties of the general trapezoidal test plate (Fig. 3a)

\begin{tabular}{ll}
\hline Geometric planform & $\theta_{1}=29.985^{\circ}, \theta_{2}=11.183^{\circ}$ \\
& $a=87 \mathrm{~mm}, b=90 \mathrm{~mm}$ \\
Thickness & $h=0.98 \times 10^{-3} \mathrm{~m}$ \\
Poisson's modulus & $v=0.35$ \\
Young's modulus & $E=6.82 \times 10^{10} \mathrm{~N} / \mathrm{m}^{2}$ \\
Flexural rigidity & $D=6.1 \mathrm{Nm}$ \\
Mass density per unit volume & $\rho=2.86 \times 10^{3} \mathrm{~kg} / \mathrm{m}^{3}$
\end{tabular}

parameter reaches the highest level for $\beta=45^{\circ}$ and the lowest level for $\beta=75^{\circ}$. However, when the aspect ratio is $b / a=2$, the variation of the fundamental frequency, is similar for both simply-supported and clamped rhomboidal laminates. In this case, it is observed that the fundamental frequency has its highest value for $\beta=0^{\circ}$ in the $\mathrm{S}-\mathrm{S}-\mathrm{S}-\mathrm{S}$ laminate and for $\beta=15^{\circ}$ in the $\mathrm{C}-\mathrm{C}-\mathrm{C}-\mathrm{C}$ laminate. Then, the fundamental frequencies decrease monotonically and reach minimum values for $\beta=75^{\circ}$.

Finally, the results for F-S-F-C plates, for both aspect ratios (i.e. $b / a=1$ and 2), show similar variation of the dynamical behaviour, when $\beta$ varies from $0^{\circ}$ to $90^{\circ}$. The fundamental frequencies have minimum values for $\beta=15^{\circ}$ and increase monotonically for $\beta>15^{\circ}$. The maximum values occur at $\beta=90^{\circ}$. In conclusion, the boundary constraints and the aspect ratio b/a have significant effects on the behaviour of the fundamental frequencies with respect to the angles of fibre orientation. 

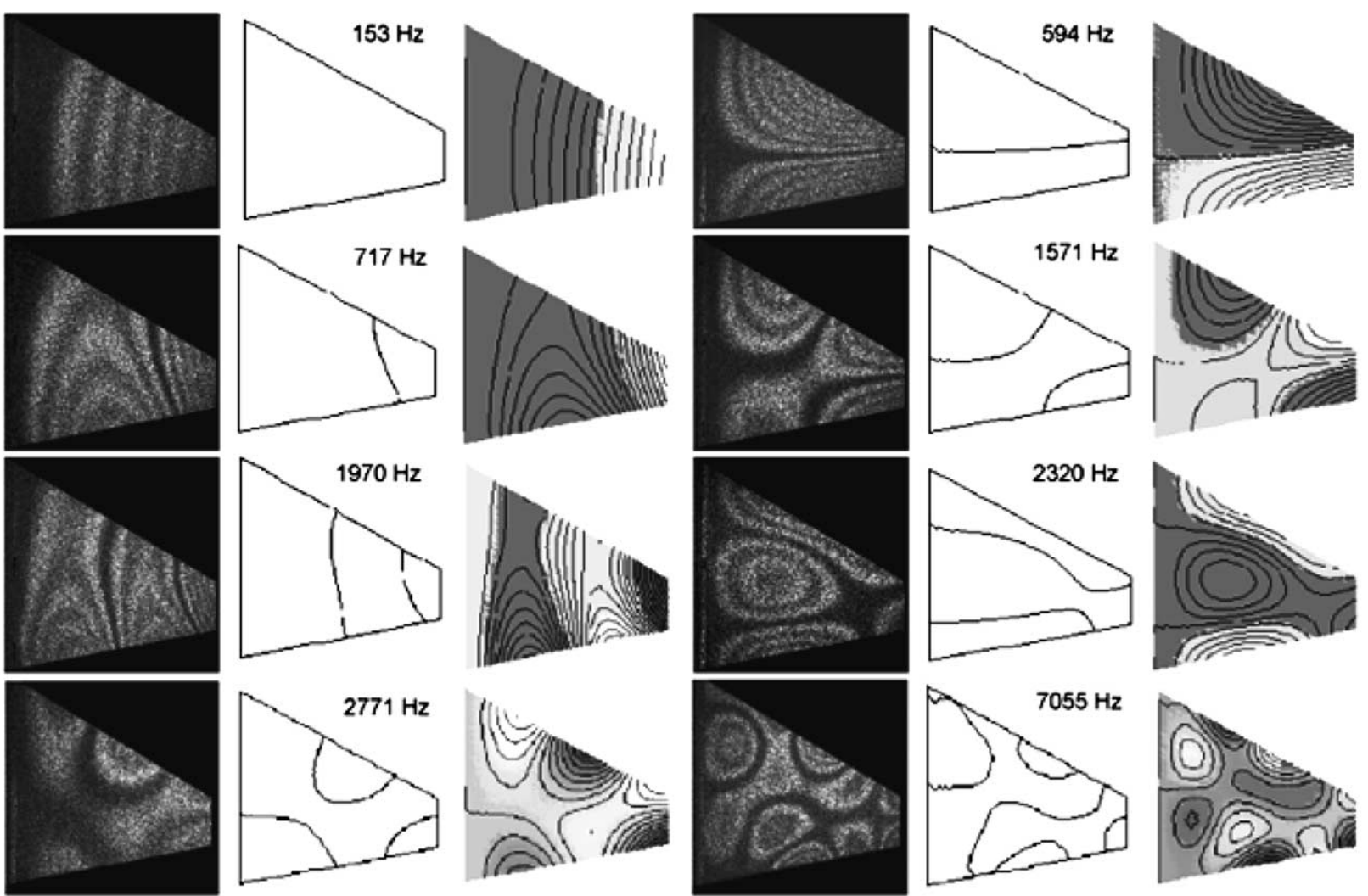

(b)
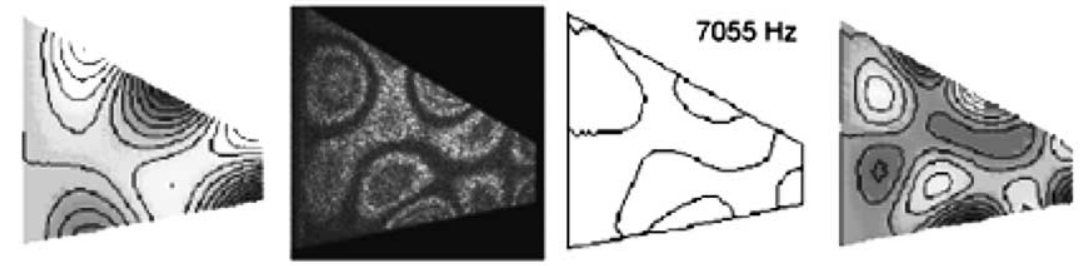

(c)

(a)

(b)

(c)

Fig. 4. Natural frequencies and modal shapes of a general trapezoidal cantilever plate. (a) Experimentally determined mode shapes [37]. (b) Nodal patterns obtained with the present method. (c) Modal shapes obtained with the present method. 
Table 6

Frequencies of free vibration, static bending deflection and bending moment for four-ply rhomboidal symmetric laminated E-glass-epoxi plates with stacking sequence $(\beta,-\beta,-\beta, \beta)$

\begin{tabular}{|c|c|c|c|c|c|c|c|c|c|c|c|}
\hline \multirow[t]{3}{*}{ b/a } & \multirow[t]{3}{*}{$\beta$} & \multirow{2}{*}{\multicolumn{2}{|c|}{ Statical analysis }} & \multicolumn{8}{|c|}{ Frequencies of free vibration $\omega a^{2} \sqrt{\rho h / D_{\beta}}$} \\
\hline & & & & \multicolumn{8}{|c|}{ Mode sequence number } \\
\hline & & $\overline{\left.\frac{W D_{\beta}}{q a^{4}}\right|_{A}}$ & $\left.\frac{M_{X}}{q a^{2}}\right|_{A}$ & 1 & 2 & 3 & 4 & 5 & 6 & 7 & 8 \\
\hline \multicolumn{12}{|c|}{$S-S-S-S$} \\
\hline \multirow[t]{7}{*}{1} & $0^{\circ}$ & 0.00133693 & 0.02376707 & 32.8576 & 75.4172 & 83.8503 & 128.528 & 154.777 & 156.297 & 200.106 & 223.860 \\
\hline & $15^{\circ}$ & 0.00141395 & 0.02596733 & 32.0502 & 72.4077 & 84.9172 & 124.163 & 146.326 & 164.154 & 192.890 & 222.625 \\
\hline & $30^{\circ}$ & 0.00147775 & 0.02567914 & 31.3968 & 71.2486 & 85.9382 & 125.305 & 139.825 & 170.808 & 194.618 & 221.936 \\
\hline & $45^{\circ}$ & 0.00150428 & 0.02315079 & 31.0589 & 70.9395 & 86.0245 & 126.585 & 136.897 & 173.601 & 194.546 & 220.311 \\
\hline & $60^{\circ}$ & 0.00146325 & 0.01892369 & 31.3628 & 71.8190 & 85.0756 & 125.927 & 139.761 & 171.037 & 193.214 & 222.200 \\
\hline & $75^{\circ}$ & 0.00139288 & 0.01536088 & 32.0320 & 73.0827 & 83.9750 & 124.846 & 146.359 & 164.213 & 191.461 & 222.520 \\
\hline & $90^{\circ}$ & 0.00132177 & 0.01526389 & 32.8607 & 75.9610 & 83.1856 & 129.063 & 154.325 & 156.608 & 199.122 & 224.404 \\
\hline \multirow[t]{7}{*}{2} & $0^{\circ}$ & 0.00366604 & 0.04754341 & 20.0737 & 36.6949 & 56.2655 & 60.6374 & 78.8026 & 92.0729 & 104.949 & 121.790 \\
\hline & $15^{\circ}$ & 0.00374594 & 0.04962897 & 19.9335 & 36.3469 & 55.3904 & 61.1931 & 77.2267 & 92.0566 & 102.409 & 121.772 \\
\hline & $30^{\circ}$ & 0.00414294 & 0.04882469 & 19.0271 & 36.1031 & 55.4023 & 59.1633 & 78.3892 & 89.6005 & 104.473 & 117.161 \\
\hline & $45^{\circ}$ & 0.00464907 & 0.04576604 & 17.9583 & 35.7434 & 53.4624 & 57.7583 & 79.7583 & 86.0722 & 107.282 & 110.188 \\
\hline & $60^{\circ}$ & 0.00500802 & 0.04042163 & 17.2236 & 35.5353 & 50.1564 & 57.6387 & 81.1768 & 82.9676 & 102.106 & 110.623 \\
\hline & $75^{\circ}$ & 0.00508757 & 0.03561888 & 16.9929 & 35.4883 & 47.8736 & 57.9540 & 80.6951 & 82.7449 & 96.1798 & 113.446 \\
\hline & $90^{\circ}$ & 0.00469514 & 0.03581890 & 17.6523 & 36.0199 & 49.6827 & 58.5220 & 82.5358 & 84.1560 & 98.5616 & 116.217 \\
\hline \multicolumn{12}{|c|}{$C-C-C-C$} \\
\hline \multirow[t]{7}{*}{1} & $0^{\circ}$ & 0.00042582 & 0.01158452 & 58.2454 & 112.062 & 122.379 & 174.548 & 205.928 & 207.868 & 257.268 & 283.315 \\
\hline & $15^{\circ}$ & 0.00042979 & 0.01215455 & 58.0819 & 108.785 & 125.147 & 170.721 & 196.149 & 218.795 & 250.273 & 283.948 \\
\hline & $30^{\circ}$ & 0.00041664 & 0.01118826 & 58.8243 & 108.225 & 128.858 & 174.302 & 189.541 & 229.261 & 253.863 & 286.712 \\
\hline & $45^{\circ}$ & 0.00040787 & 0.00963926 & 59.1970 & 108.212 & 130.331 & 177.483 & 185.940 & 234.419 & 254.254 & 285.375 \\
\hline & $60^{\circ}$ & 0.00040945 & 0.00807797 & 58.7931 & 108.550 & 128.284 & 175.028 & 188.858 & 230.784 & 251.345 & 288.012 \\
\hline & $75^{\circ}$ & 0.00041705 & 0.00704269 & 58.0039 & 109.391 & 124.142 & 171.430 & 195.568 & 219.788 & 248.017 & 283.799 \\
\hline & $90^{\circ}$ & 0.00041507 & 0.00737193 & 58.1653 & 112.883 & 121.239 & 175.096 & 205.294 & 208.253 & 255.949 & 283.539 \\
\hline \multirow[t]{7}{*}{2} & $0^{\circ}$ & 0.00111174 & 0.02207262 & 36.8314 & 58.8535 & 83.1270 & 87.6450 & 109.901 & 125.523 & 140.416 & 159.182 \\
\hline & $15^{\circ}$ & 0.00110060 & 0.02254566 & 37.0481 & 58.5976 & 82.0608 & 89.1788 & 108.280 & 125.692 & 137.699 & 159.292 \\
\hline & $30^{\circ}$ & 0.00115863 & 0.02137345 & 36.0536 & 57.8697 & 81.2752 & 87.4305 & 109.087 & 122.138 & 139.372 & 154.232 \\
\hline & $45^{\circ}$ & 0.00126541 & 0.01952095 & 34.4103 & 56.7976 & 78.8899 & 84.7655 & 109.949 & 117.102 & 141.701 & 146.240 \\
\hline & $60^{\circ}$ & 0.00140589 & 0.01768004 & 32.5536 & 55.6541 & 74.3086 & 82.9053 & 110.424 & 112.245 & 135.837 & 144.122 \\
\hline & $75^{\circ}$ & 0.00152877 & 0.01651642 & 31.1404 & 54.7190 & 70.1211 & 81.9192 & 108.584 & 110.705 & 127.178 & 145.972 \\
\hline & $90^{\circ}$ & 0.00146509 & 0.01713289 & 31.8310 & 55.4846 & 72.0156 & 82.6599 & 110.932 & 112.336 & 129.614 & 149.142 \\
\hline
\end{tabular}


Table 6 (continued)

\begin{tabular}{|c|c|c|c|c|c|c|c|c|c|c|c|}
\hline \multirow[t]{3}{*}{$\overline{b / a}$} & \multirow[t]{3}{*}{$\beta$} & \multirow{2}{*}{\multicolumn{2}{|c|}{ Statical analysis }} & \multicolumn{8}{|c|}{ Frequencies of free vibration $\omega a^{2} \sqrt{\rho h / D_{\beta}}$} \\
\hline & & & & \multicolumn{8}{|c|}{ Mode sequence number } \\
\hline & & $\overline{\left.\frac{W D_{\beta}}{q a^{4}}\right|_{A}}$ & $\left.\frac{M_{x}}{q a^{2}}\right|_{A}$ & 1 & 2 & 3 & 4 & 5 & 6 & 7 & 8 \\
\hline \multicolumn{12}{|c|}{$F-S-F-C$} \\
\hline \multirow[t]{7}{*}{1} & $0^{\circ}$ & 0.00192178 & 0.02097692 & 20.0749 & 35.9246 & 60.7807 & 69.0070 & 98.0648 & 116.767 & 129.733 & 141.435 \\
\hline & $15^{\circ}$ & 0.00222384 & 0.01947506 & 18.8546 & 33.9168 & 57.7059 & 67.2526 & 91.9433 & 113.083 & 126.054 & 138.325 \\
\hline & $30^{\circ}$ & 0.00227301 & 0.01482298 & 18.9978 & 32.3254 & 58.0007 & 65.9827 & 89.2559 & 114.376 & 125.899 & 134.964 \\
\hline & $45^{\circ}$ & 0.00224813 & 0.01482298 & 19.3527 & 31.2364 & 59.3839 & 64.1125 & 88.4524 & 117.130 & 123.865 & 132.590 \\
\hline & $60^{\circ}$ & 0.00221066 & 0.01122682 & 19.7787 & 31.2895 & 61.3389 & 62.7327 & 89.3265 & 118.306 & 123.680 & 131.693 \\
\hline & $75^{\circ}$ & 0.00208758 & 0.01000555 & 20.5661 & 32.2784 & 62.3017 & 64.2770 & 91.8491 & 115.788 & 128.965 & 132.427 \\
\hline & $90^{\circ}$ & 0.00180005 & 0.01006510 & 22.0410 & 34.4608 & 64.0139 & 68.5484 & 97.9682 & 117.646 & 133.655 & 138.400 \\
\hline \multirow[t]{7}{*}{2} & $0^{\circ}$ & 0.00575835 & 0.05424203 & 8.51802 & 19.2463 & 25.8933 & 38.1105 & 43.7022 & 57.8248 & 63.5934 & 75.7244 \\
\hline & $15^{\circ}$ & 0.00671898 & 0.05168905 & 8.17785 & 18.5432 & 24.8294 & 37.3132 & 41.8714 & 56.5665 & 60.9715 & 72.8666 \\
\hline & $30^{\circ}$ & 0.00733401 & 0.04091217 & 8.36055 & 18.2451 & 24.8001 & 37.4033 & 41.7509 & 57.7187 & 58.9622 & 72.4577 \\
\hline & $45^{\circ}$ & 0.00768447 & 0.03488778 & 8.54956 & 17.7511 & 25.1077 & 36.9631 & 42.2672 & 55.9722 & 60.5213 & 71.9866 \\
\hline & $60^{\circ}$ & 0.00802293 & 0.03481320 & 8.62899 & 17.3105 & 25.6321 & 36.0528 & 43.2175 & 54.2805 & 62.6014 & 71.6312 \\
\hline & $75^{\circ}$ & 0.00813069 & 0.03593550 & 8.75546 & 17.3887 & 26.4330 & 35.4586 & 44.6321 & 54.0893 & 63.2167 & 73.3099 \\
\hline & $90^{\circ}$ & 0.00706228 & 0.03732225 & 9.19851 & 18.5509 & 27.6271 & 36.8327 & 46.5567 & 57.2937 & 63.6317 & 77.1701 \\
\hline
\end{tabular}


$\left(30^{\circ},-30^{\circ},-30^{\circ}, 30\right)$
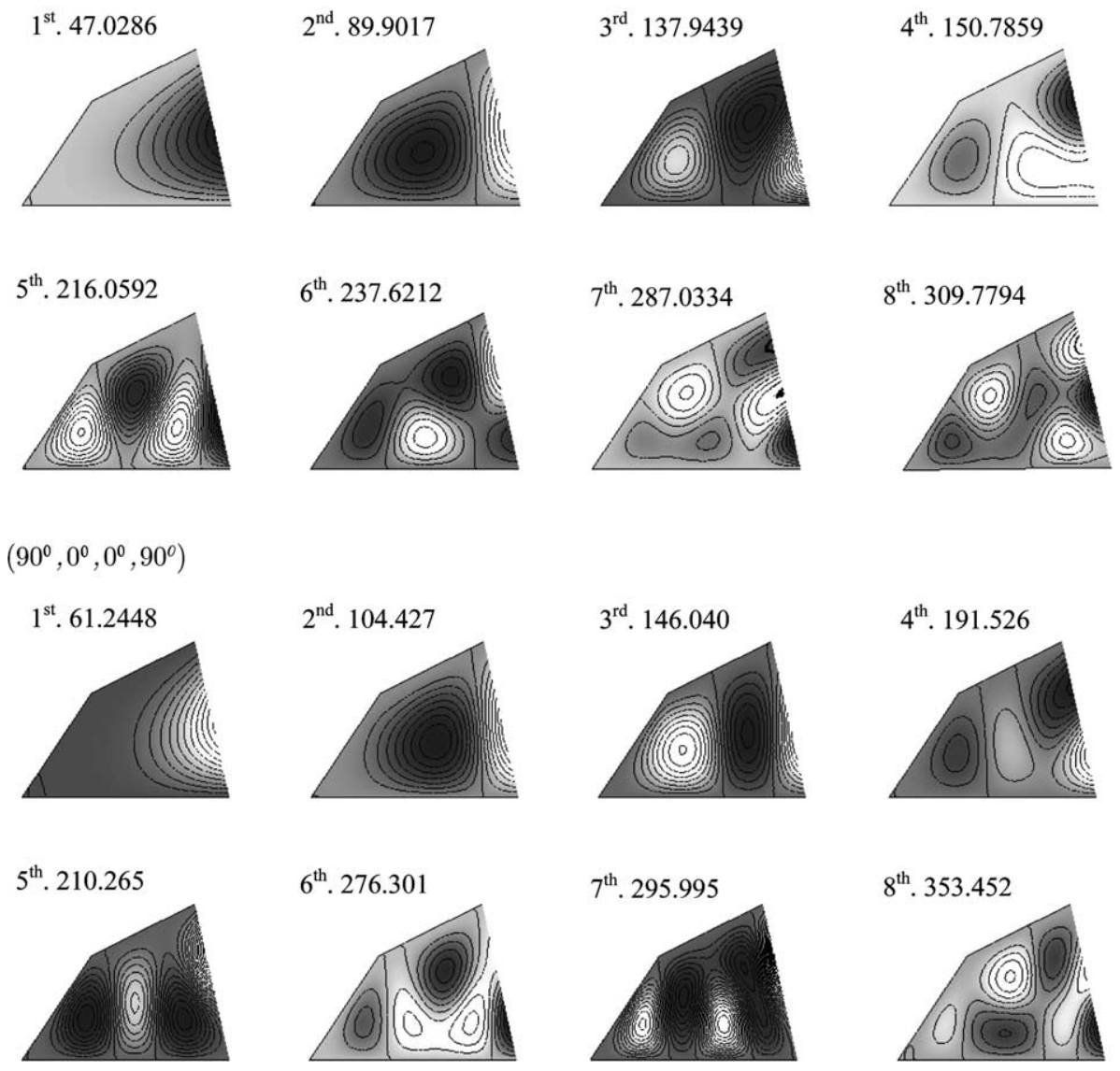

Fig. 5. Transverse vibration frequencies $\omega a^{2} \sqrt{\rho h / D_{\beta}}$ and mode shapes of a S-F-C-S general quadrilateral E-glass/epoxi laminate.

\subsection{Other quadrilateral plates}

The developed Ritz formulation has been further applied to generate results for laminated E-glass/epoxi plates with general quadrilateral planforms (see Fig. 3d). The presented results correspond to a angle-ply laminate with stacking sequence $\left(30^{\circ},-30^{\circ},-30^{\circ}, 30^{\circ}\right)$ and to a cross-ply laminate with stacking sequence $\left(90^{\circ}, 0^{\circ}, 0^{\circ}, 90^{\circ}\right)$ and aspect ratio $b / a=1 / 2$, for both cases. The plates are simply supported on edges (1) and (4), free on edge (2) and clamped on edge (3). Fig. 5 shows the first eight non-dimensional free vibration frequencies $\omega a^{2} \sqrt{\rho h / D_{\beta}}$ and their corresponding modal shapes. It is observed that the frequency parameters are higher for the cross-ply laminate than for the angle-ply laminate. There exist a little difference between the modal shapes for both cases when the first three natural frequencies are considered.

\section{Conclusions}

A Ritz approach has been developed for the study of the dynamical and statical behaviour of symmetrically laminated composite plates. The proposed method is based on the classical laminated plate theory and 
uses natural coordinates to express the geometry of different laminates in a simple form. The deflection of the plate is approximated by a set of beam characteristic orthogonal polynomials generated using the GramSchmidt procedure. The algorithm developed is very general and allowed us to take into account a great variety of geometrical shapes, material properties and combinations of classical boundary conditions.

Numerical applications include trapezoidal, skew, rhomboidal and general quadrilateral laminates. For the dynamical analysis frequencies and modal shapes of free vibration have been obtained, and for the statical analysis transverse deflections and bending moments have been determined. For trapezoidal and skew plates, very close agreement was found between the present results and the comparative solutions. Besides, all applications demonstrate that the present technique is accurate and efficient. Consequently it constitutes an efficient tool for the determination of natural frequencies and static deflections in an important number of plate problems, and it is of interest in design works.

\section{Acknowledgement}

The authors are indebted to the reviewers of the paper for their constructive comments and suggestions. The present study has been partially sponsored by the Consejo de Investigación, Proyecto CIUNSA 1229.

\section{Appendix A. Coordinate transformation}

The definition of the transformation matrixes in Eq. (10), which describe the relation between the derivatives with respect to the Cartesian coordinates $(x, y)$ and the derivatives with respect to the natural coordinates $(\xi, \eta)$, are obtained applying successively the rule of derivation of composite functions and are given by

$$
\left[O p^{(1)}\right]=\left[\begin{array}{ccc}
a_{1}^{\prime} & a_{2}^{\prime} & -a_{3}^{\prime} \\
b_{1}^{\prime} & b_{2}^{\prime} & -b_{3}^{\prime} \\
-c_{1}^{\prime} & -c_{2}^{\prime} & c_{3}^{\prime}
\end{array}\right], \quad\left[O p^{(2)}\right]=\left[\begin{array}{cc}
\sum_{i=1}^{3} a_{i}^{\prime} \alpha_{i}^{\prime} & \sum_{i=1}^{3} a_{i}^{\prime} \beta_{i}^{\prime} \\
\sum_{i=1}^{3} b_{i}^{\prime} \alpha_{i}^{\prime} & \sum_{i=1}^{3} b_{i}^{\prime} \beta_{i}^{\prime} \\
-\sum_{i=1}^{3} c_{i}^{\prime} \alpha_{i}^{\prime} & -\sum_{i=1}^{3} c_{i}^{\prime} \beta_{i}^{\prime}
\end{array}\right]
$$

where

$$
\begin{aligned}
& a_{1}^{\prime}=\frac{J_{22}^{2}}{|\mathbf{J}|^{2}}, \quad a_{2}^{\prime}=\frac{J_{12}^{2}}{|\mathbf{J}|^{2}}, \quad a_{3}^{\prime}=2 \frac{J_{12} J_{22}}{|\mathbf{J}|^{2}}, \\
& b_{1}^{\prime}=\frac{J_{21}^{2}}{|\mathbf{J}|^{2}}, \quad b_{2}^{\prime}=\frac{J_{11}^{2}}{|\mathbf{J}|^{2}}, \quad b_{3}^{\prime}=2 \frac{J_{11} J_{21}}{|\mathbf{J}|^{2}}, \\
& c_{1}^{\prime}=\frac{J_{21} J_{22}}{|\mathbf{J}|^{2}}, \quad c_{2}^{\prime}=\frac{J_{11} J_{12}}{|\mathbf{J}|^{2}}, \quad c_{3}^{\prime}=\frac{J_{11} J_{22}+J_{12} J_{21}}{|\mathbf{J}|^{2}}, \\
& \alpha_{1}^{\prime}=\frac{-J_{11, \xi} J_{22}+J_{12, \xi} J_{21}}{|\mathbf{J}|}, \quad \alpha_{2}^{\prime}=\frac{-J_{21, \eta} J_{22}+J_{22, \eta} J_{21}}{|\mathbf{J}|}, \quad \alpha_{3}^{\prime}=\frac{J_{11, \eta} J_{22}-J_{22, \xi} J_{21}}{|\mathbf{J}|}, \\
& \beta_{1}^{\prime}=\frac{J_{11, \xi} J_{12}-J_{12, \xi} J_{11}}{|\mathbf{J}|}, \quad \beta_{2}^{\prime}=\frac{J_{21, \eta} J_{12}-J_{22, \eta} J_{11}}{|\mathbf{J}|}, \quad \beta_{3}^{\prime}=\frac{J_{11, \eta} J_{12}-J_{22, \xi} J_{11}}{|\mathbf{J}|},
\end{aligned}
$$




$$
\mathbf{J}_{2}=\left[\begin{array}{lll}
\left(\frac{\partial x}{\partial \xi}\right)^{2} & \left(\frac{\partial y}{\partial \xi}\right)^{2} & 2 \frac{\partial x}{\partial \xi} \frac{\partial y}{\partial \xi} \\
\left(\frac{\partial x}{\partial \eta}\right)^{2} & \left(\frac{\partial y}{\partial \eta}\right)^{2} & 2 \frac{\partial x}{\partial \eta} \frac{\partial y}{\partial \eta} \\
\frac{\partial x}{\partial \xi} \frac{\partial x}{\partial \eta} & \frac{\partial y}{\partial \xi} \frac{\partial y}{\partial \eta} & \frac{\partial x}{\partial \xi} \frac{\partial y}{\partial \eta}+\frac{\partial x}{\partial \eta} \frac{\partial y}{\partial \xi}
\end{array}\right]=\left[\begin{array}{ccc}
J_{11}^{2} & J_{12}^{2} & 2 J_{12} J_{11} \\
J_{21}^{2} & J_{22}^{2} & 2 J_{21} J_{22} \\
J_{11} J_{21} & J_{12} J_{22} & J_{11} J_{22}+J_{21} J_{12}
\end{array}\right]
$$

and $|\mathbf{J}|$ denotes the Jacobian determinant.

\section{Appendix B. Definitions of functions $S_{i}$ in Eq. (13)}

After substitution of Eq. (10) into Eq. (5) one obtains the maximum strain energy as a function of the derivatives of the displacement $W$ with respect to the natural coordinates $\xi$, $\eta$. The factors of these derivatives depend on the geometrical and mechanical characteristics of the plates, and are given by

$$
\begin{aligned}
& S_{1}(\xi, \eta)=D_{11} a_{1}^{\prime 2}+D_{22} b_{1}^{\prime 2}+2 D_{12} a_{1}^{\prime} b_{1}^{\prime}+4 D_{66} c_{1}^{\prime 2}-4 D_{16} a_{1}^{\prime} c_{1}^{\prime}-4 D_{26} b_{1}^{\prime} c_{1}^{\prime}, \\
& S_{2}(\xi, \eta)=D_{11} a_{2}^{\prime 2}+D_{22} b_{2}^{\prime 2}+2 D_{12} a_{2}^{\prime} b_{2}^{\prime}+4 D_{66} c_{2}^{\prime 2}-4 D_{16} a_{2}^{\prime} c_{2}^{\prime}-4 D_{26} b_{2}^{\prime} c_{2}^{\prime}, \\
& S_{3}(\xi, \eta)=2 D_{11} a_{1}^{\prime} a_{2}^{\prime}+2 D_{22} b_{1}^{\prime} b_{2}^{\prime}+2 D_{12}\left(b_{1}^{\prime} a_{2}^{\prime}+b_{2}^{\prime} a_{1}^{\prime}\right)+8 D_{66} c_{1}^{\prime} c_{2}^{\prime}-4 D_{16}\left(c_{2}^{\prime} a_{1}^{\prime}+c_{1}^{\prime} a_{2}^{\prime}\right)-4 D_{26}\left(b_{2}^{\prime} c_{1}^{\prime}+b_{1}^{\prime} c_{2}^{\prime}\right), \\
& S_{4}(\xi, \eta)=D_{11} a_{3}^{\prime 2}+D_{22} b_{3}^{\prime 2}+2 D_{12} a_{3}^{\prime} b_{3}^{\prime}+4 D_{66} c_{3}^{\prime 2}-4 D_{16} a_{3}^{\prime} c_{3}^{\prime}-4 D_{26} b_{3}^{\prime} c_{3}^{\prime}, \\
& S_{5}(\xi, \eta)=-2 D_{11} a_{3}^{\prime} a_{1}^{\prime}-2 D_{22} b_{3}^{\prime} b_{1}^{\prime}-2 D_{12}\left(b_{1}^{\prime} a_{3}^{\prime}+b_{3}^{\prime} a_{1}^{\prime}\right)-8 D_{66} c_{3}^{\prime} c_{1}^{\prime}+4 D_{16}\left(a_{1}^{\prime} c_{3}^{\prime}+c_{1}^{\prime} a_{3}^{\prime}\right)+4 D_{26}\left(b_{3}^{\prime} c_{1}^{\prime}+b_{1}^{\prime} c_{3}^{\prime}\right), \\
& S_{6}(\xi, \eta)=-2 D_{11} a_{2}^{\prime} a_{3}^{\prime}-2 D_{22} b_{3}^{\prime} b_{2}^{\prime}-2 D_{12}\left(b_{3}^{\prime} a_{2}^{\prime}+b_{2}^{\prime} a_{3}^{\prime}\right)-8 D_{66} c_{3}^{\prime} c_{2}^{\prime}+4 D_{16}\left(c_{3}^{\prime} a_{2}^{\prime}+c_{2}^{\prime} a_{3}^{\prime}\right)+4 D_{26}\left(b_{3}^{\prime} c_{2}^{\prime}+b_{2}^{\prime} c_{3}^{\prime}\right), \\
& S_{7}(\xi, \eta)=2 D_{11} a_{1}^{\prime} \sum_{i=1}^{3} a_{i}^{\prime} \alpha_{i}^{\prime}+2 D_{22} b_{1}^{\prime} \sum_{i=1}^{3} b_{i}^{\prime} \alpha_{i}^{\prime}+2 D_{12}\left(a_{1}^{\prime} \sum_{i=1}^{3} b_{i}^{\prime} \alpha_{i}^{\prime}+b_{1}^{\prime} \sum_{i=1}^{3} a_{i}^{\prime} \alpha_{i}^{\prime}\right) \\
& +8 D_{66} c_{1}^{\prime} \sum_{i=1}^{3} c_{i}^{\prime} \alpha_{i}^{\prime}-4 D_{16}\left(a_{1}^{\prime} \sum_{i=1}^{3} c_{i}^{\prime} \alpha_{i}^{\prime}+c_{1}^{\prime} \sum_{i=1}^{3} a_{i}^{\prime} \alpha_{i}^{\prime}\right)-4 D_{26}\left(b_{1}^{\prime} \sum_{i=1}^{3} c_{i}^{\prime} \alpha_{i}^{\prime}+c_{1}^{\prime} \sum_{i=1}^{3} b_{i}^{\prime} \alpha_{i}^{\prime}\right), \\
& S_{8}(\xi, \eta)=2 D_{11} a_{2}^{\prime} \sum_{i=1}^{3} a_{i}^{\prime} \beta_{i}^{\prime}+2 D_{22} b_{2}^{\prime} \sum_{i=1}^{3} b_{i}^{\prime} \beta_{i}^{\prime}+2 D_{12}\left(a_{2}^{\prime} \sum_{i=1}^{3} b_{i}^{\prime} \beta_{i}^{\prime}+b_{2}^{\prime} \sum_{i=1}^{3} a_{i}^{\prime} \beta_{i}^{\prime}\right) \\
& +8 D_{66} c_{2}^{\prime} \sum_{i=1}^{3} c_{i}^{\prime} \beta_{i}^{\prime}-4 D_{16}\left(a_{2}^{\prime} \sum_{i=1}^{3} c_{i}^{\prime} \beta_{i}^{\prime}+c_{2}^{\prime} \sum_{i=1}^{3} a_{i}^{\prime} \beta_{i}^{\prime}\right)-4 D_{26}\left(c_{2}^{\prime} \sum_{i=1}^{3} b_{i}^{\prime} \beta_{i}^{\prime}+b_{2}^{\prime} \sum_{i=1}^{3} c_{i}^{\prime} \beta_{i}^{\prime}\right), \\
& S_{9}(\xi, \eta)=2 D_{11} a_{1}^{\prime} \sum_{i=1}^{3} a_{i}^{\prime} \beta_{i}^{\prime}+2 D_{22} b_{1}^{\prime} \sum_{i=1}^{3} b_{i}^{\prime} \beta_{i}^{\prime}+2 D_{12}\left(a_{1}^{\prime} \sum_{i=1}^{3} b_{i}^{\prime} \beta_{i}^{\prime}+b_{1}^{\prime} \sum_{i=1}^{3} a_{i}^{\prime} \beta_{i}^{\prime}\right) \\
& +8 D_{66} c_{1}^{\prime} \sum_{i=1}^{3} c_{i}^{\prime} \beta_{i}^{\prime}-4 D_{16}\left(a_{1}^{\prime} \sum_{i=1}^{3} c_{i}^{\prime} \beta_{i}^{\prime}+c_{1}^{\prime} \sum_{i=1}^{3} a_{i}^{\prime} \beta_{i}^{\prime}\right)-4 D_{26}\left(c_{1}^{\prime} \sum_{i=1}^{3} b_{i}^{\prime} \beta_{i}^{\prime}+b_{1}^{\prime} \sum_{i=1}^{3} c_{i}^{\prime} \beta_{i}^{\prime}\right),
\end{aligned}
$$




$$
\begin{aligned}
& S_{10}(\xi, \eta)=2 D_{11} a_{2}^{\prime} \sum_{i=1}^{3} a_{i}^{\prime} \alpha_{i}^{\prime}+2 D_{22} b_{2}^{\prime} \sum_{i=1}^{3} b_{i}^{\prime} \alpha_{i}^{\prime}+2 D_{12}\left(a_{2}^{\prime} \sum_{i=1}^{3} b_{i}^{\prime} \alpha_{i}^{\prime}+b_{2}^{\prime} \sum_{i=1}^{3} a_{i}^{\prime} \alpha_{i}^{\prime}\right) \\
& +8 D_{66} c_{2}^{\prime} \sum_{i=1}^{3} c_{i}^{\prime} \alpha_{i}^{\prime}-4 D_{16}\left(a_{2}^{\prime} \sum_{i=1}^{3} c_{i}^{\prime} \alpha_{i}^{\prime}+c_{2}^{\prime} \sum_{i=1}^{3} a_{i}^{\prime} \alpha_{i}^{\prime}\right)-4 D_{26}\left(c_{2}^{\prime} \sum_{i=1}^{3} b_{i}^{\prime} \alpha_{i}^{\prime}+b_{2}^{\prime} \sum_{i=1}^{3} c_{i}^{\prime} \alpha_{i}^{\prime}\right), \\
& S_{11}(\xi, \eta)=-2 D_{11} a_{3}^{\prime} \sum_{i=1}^{3} a_{i}^{\prime} \alpha_{i}^{\prime}-2 D_{22} b_{3}^{\prime} \sum_{i=1}^{3} b_{i}^{\prime} \alpha_{i}^{\prime}-2 D_{12}\left(a_{3}^{\prime} \sum_{i=1}^{3} b_{i}^{\prime} \alpha_{i}^{\prime}+b_{3}^{\prime} \sum_{i=1}^{3} a_{i}^{\prime} \alpha_{i}^{\prime}\right) \\
& -8 D_{66} c_{3}^{\prime} \sum_{i=1}^{3} c_{i}^{\prime} \alpha_{i}^{\prime}+4 D_{16}\left(a_{3}^{\prime} \sum_{i=1}^{3} c_{i}^{\prime} \alpha_{i}^{\prime}+c_{3}^{\prime} \sum_{i=1}^{3} a_{i}^{\prime} \alpha_{i}^{\prime}\right)+4 D_{26}\left(c_{3}^{\prime} \sum_{i=1}^{3} b_{i}^{\prime} \alpha_{i}^{\prime}+b_{3}^{\prime} \sum_{i=1}^{3} c_{i}^{\prime} \alpha_{i}^{\prime}\right), \\
& S_{12}(\xi, \eta)=-2 D_{11} a_{3}^{\prime} \sum_{i=1}^{3} a_{i}^{\prime} \beta_{i}^{\prime}-2 D_{22} b_{3}^{\prime} \sum_{i=1}^{3} b_{i}^{\prime} \beta_{i}^{\prime}-2 D_{12}\left(a_{3}^{\prime} \sum_{i=1}^{3} b_{i}^{\prime} \beta_{i}^{\prime}+b_{3}^{\prime} \sum_{i=1}^{3} a_{i}^{\prime} \beta_{i}^{\prime}\right) \\
& -8 D_{66} c_{3}^{\prime} \sum_{i=1}^{3} c_{i}^{\prime} \beta_{i}^{\prime}+4 D_{16}\left(a_{3}^{\prime} \sum_{i=1}^{3} c_{i}^{\prime} \beta_{i}^{\prime}+c_{3}^{\prime} \sum_{i=1}^{3} a_{i}^{\prime} \beta_{i}^{\prime}\right)+4 D_{26}\left(c_{3}^{\prime} \sum_{i=1}^{3} b_{i}^{\prime} \beta_{i}^{\prime}+b_{3}^{\prime} \sum_{i=1}^{3} c_{i}^{\prime} \beta_{i}^{\prime}\right) \\
& S_{13}(\xi, \eta)=D_{11}\left(\sum_{i=1}^{3} a_{i}^{\prime} \alpha_{i}^{\prime}\right)^{2}+D_{22}\left(\sum_{i=1}^{3} b_{i}^{\prime} \alpha_{i}^{\prime}\right)^{2}+2 D_{12} \sum_{i=1}^{3} b_{i}^{\prime} \alpha_{i}^{\prime} \sum_{i=1}^{3} a_{i}^{\prime} \alpha_{i}^{\prime} \\
& +4 D_{66}\left(\sum_{i=1}^{3} c_{i}^{\prime} \alpha_{i}^{\prime}\right)^{2}-4 D_{16} \sum_{i=1}^{3} c_{i}^{\prime} \alpha_{i}^{\prime} \sum_{i=1}^{3} a_{i}^{\prime} \alpha_{i}^{\prime}-4 D_{26} \sum_{i=1}^{3} b_{i}^{\prime} \alpha_{i}^{\prime} \sum_{i=1}^{3} c_{i}^{\prime} \alpha_{i}^{\prime}, \\
& S_{14}(\xi, \eta)=D_{11}\left(\sum_{i=1}^{3} a_{i}^{\prime} \beta_{i}^{\prime}\right)^{2}+D_{22}\left(\sum_{i=1}^{3} b_{i}^{\prime} \beta_{i}^{\prime}\right)^{2}+2 D_{12} \sum_{i=1}^{3} b_{i}^{\prime} \beta_{i}^{\prime} \sum_{i=1}^{3} a_{i}^{\prime} \beta_{i}^{\prime} \\
& +4 D_{66}\left(\sum_{i=1}^{3} c_{i}^{\prime} \beta_{i}^{\prime}\right)^{2}-4 D_{16} \sum_{i=1}^{3} c_{i}^{\prime} \beta_{i}^{\prime} \sum_{i=1}^{3} a_{i}^{\prime} \beta_{i}^{\prime}-4 D_{26} \sum_{i=1}^{3} b_{i}^{\prime} \beta_{i}^{\prime} \sum_{i=1}^{3} c_{i}^{\prime} \beta_{i}^{\prime}, \\
& S_{15}(\xi, \eta)=2 D_{11} \sum_{i=1}^{3} a_{i}^{\prime} \alpha_{i}^{\prime} \sum_{i=1}^{3} a_{i}^{\prime} \beta_{i}^{\prime}+2 D_{22} \sum_{i=1}^{3} b_{i}^{\prime} \alpha_{i}^{\prime} \sum_{i=1}^{3} b_{i}^{\prime} \beta_{i}^{\prime}+2 D_{12}\left(\sum_{i=1}^{3} b_{i}^{\prime} \beta_{i}^{\prime} \sum_{i=1}^{3} a_{i}^{\prime} \alpha_{i}^{\prime}+\sum_{i=1}^{3} b_{i}^{\prime} \alpha_{i}^{\prime} \sum_{i=1}^{3} a_{i}^{\prime} \beta_{i}^{\prime}\right) \\
& +8 D_{66} \sum_{i=1}^{3} c_{i}^{\prime} \alpha_{i}^{\prime} \sum_{i=1}^{3} c_{i}^{\prime} \beta_{i}^{\prime}-4 D_{16}\left(\sum_{i=1}^{3} c_{i}^{\prime} \beta_{i}^{\prime} \sum_{i=1}^{3} a_{i}^{\prime} \alpha_{i}^{\prime}+\sum_{i=1}^{3} c_{i}^{\prime} \alpha_{i}^{\prime} \sum_{i=1}^{3} a_{i}^{\prime} \beta_{i}^{\prime}\right) \\
& -4 D_{26}\left(\sum_{i=1}^{3} b_{i}^{\prime} \beta_{i}^{\prime} \sum_{i=1}^{3} c_{i}^{\prime} \alpha_{i}^{\prime}+\sum_{i=1}^{3} b_{i}^{\prime} \alpha_{i}^{\prime} \sum_{i=1}^{3} c_{i}^{\prime} \beta_{i}^{\prime}\right)
\end{aligned}
$$

where $D_{i j}(i, j=1,2,6)$ are the conventional laminate stiffness coefficients and $a_{i}^{\prime}, b_{i}^{\prime}, c_{i}^{\prime}, \alpha_{i}^{\prime}, \beta_{i}^{\prime},(i=1, \ldots, 3)$ are defined in Appendix A. 


\section{Appendix C. Minimisation of energy functionals}

In this Appendix the minimisation of the energy functionals as given in equations (18) and (19) are detailed.

For minimisation purpose, first we replace the approximating function (15) into the expression of $U_{\max }$ given by Eq. (13), as follows

$$
\begin{aligned}
& U_{\max }=\frac{1}{2} \int_{-1}^{1} \int_{-1}^{1}\left\{S_{1}\left(\sum_{i, j=1}^{M, N} c_{i j} \frac{\mathrm{d}^{2} p_{i}(\xi)}{\mathrm{d} \xi^{2}} q_{j}(\eta)\right)^{2}+S_{2}\left(\sum_{i, j=1}^{M, N} c_{i j} p_{i}(\xi) \frac{\mathrm{d}^{2} q_{j}(\eta)}{\mathrm{d} \eta^{2}}\right)^{2}\right. \\
& +S_{3}\left(\sum_{i, j=1}^{M, N} c_{i j} \frac{\mathrm{d}^{2} p_{i}(\xi)}{\mathrm{d} \xi^{2}} q_{j}(\eta)\right)\left(\sum_{i, j=1}^{M, N} c_{i j} p_{i}(\xi) \frac{\mathrm{d}^{2} q_{j}(\eta)}{\mathrm{d} \eta^{2}}\right) \\
& +S_{4}\left(\sum_{i, j=1}^{M, N} c_{i j} \frac{\mathrm{d} p_{i}(\xi)}{\mathrm{d} \xi} \frac{\mathrm{d} q_{j}(\eta)}{\mathrm{d} \eta}\right)^{2} \\
& +S_{5}\left(\sum_{i, j=1}^{M, N} c_{i j} \frac{\mathrm{d}^{2} p_{i}(\xi)}{\mathrm{d} \xi^{2}} q_{j}(\eta)\right)\left(\sum_{i, j=1}^{M, N} c_{i j} \frac{\mathrm{d} p_{i}(\xi)}{\mathrm{d} \xi} \frac{\mathrm{d} q_{j}(\eta)}{\mathrm{d} \eta}\right) \\
& +S_{6}\left(\sum_{i, j=1}^{M, N} c_{i j} p_{i}(\xi) \frac{\mathrm{d}^{2} q_{j}(\eta)}{\mathrm{d} \eta^{2}}\right)\left(\sum_{i, j=1}^{M, N} c_{i j} \frac{\mathrm{d} p_{i}(\xi)}{\mathrm{d} \xi} \frac{\mathrm{d} q_{j}(\eta)}{\mathrm{d} \eta}\right) \\
& +S_{7}\left(\sum_{i, j=1}^{M, N} c_{i j} \frac{\mathrm{d}^{2} p_{i}(\xi)}{\mathrm{d} \xi^{2}} q_{j}(\eta)\right)\left(\sum_{i, j=1}^{M, N} c_{i j} \frac{\mathrm{d} p_{i}(\xi)}{\mathrm{d} \xi} q_{j}(\eta)\right) \\
& +S_{8}\left(\sum_{i, j=1}^{M, N} c_{i j} p_{i}(\xi) \frac{\mathrm{d}^{2} q_{j}(\eta)}{\mathrm{d} \eta^{2}}\right)\left(\sum_{i, j=1}^{M, N} c_{i j} p_{i}(\xi) \frac{\mathrm{d} q_{j}(\eta)}{\mathrm{d} \eta}\right) \\
& +S_{9}\left(\sum_{i, j=1}^{M, N} c_{i j} \frac{\mathrm{d}^{2} p_{i}(\xi)}{\mathrm{d} \xi^{2}} q_{j}(\eta)\right)\left(\sum_{i, j=1}^{M, N} c_{i j} p_{i}(\xi) \frac{\mathrm{d} q_{j}(\eta)}{\mathrm{d} \eta}\right) \\
& +S_{10}\left(\sum_{i, j=1}^{M, N} c_{i j} p_{i}(\xi) \frac{\mathrm{d}^{2} q_{j}(\eta)}{\mathrm{d} \eta^{2}}\right)\left(\sum_{i, j=1}^{M, N} c_{i j} \frac{\mathrm{d} p_{i}(\xi)}{\mathrm{d} \xi} q_{j}(\eta)\right) \\
& +S_{11}\left(\sum_{i, j=1}^{M, N} c_{i j} \frac{\mathrm{d} p_{i}(\xi)}{\mathrm{d} \xi} \frac{\mathrm{d} q_{j}(\eta)}{\mathrm{d} \eta}\right)\left(\sum_{i, j=1}^{M, N} c_{i j} \frac{\mathrm{d} p_{i}(\xi)}{\mathrm{d} \xi} q_{j}(\eta)\right) \\
& +S_{12}\left(\sum_{i, j=1}^{M, N} c_{i j} \frac{\mathrm{d} p_{i}(\xi)}{\mathrm{d} \xi} \frac{\mathrm{d} q_{j}(\eta)}{\mathrm{d} \eta}\right)\left(\sum_{i, j=1}^{M, N} c_{i j} p_{i}(\xi) \frac{\mathrm{d} q_{i}(\eta)}{\mathrm{d} \eta}\right) \\
& +S_{13}\left(\sum_{i, j=1}^{M, N} c_{i j} \frac{\mathrm{d} p_{i}(\xi)}{\mathrm{d} \xi} q_{j}(\eta)\right)^{2}+S_{14}\left(\sum_{k, h=1}^{M, N} c_{k h} p_{i}(\xi) \frac{\mathrm{d} q_{j}(\eta)}{\mathrm{d} \eta}\right)^{2} \\
& \left.+S_{15}\left(\sum_{i, j=1}^{M, N} c_{i j} \frac{\mathrm{d} p_{i}(\xi)}{\mathrm{d} \xi} q_{j}(\eta)\right)\left(\sum_{i, j=1}^{M, N} c_{i j} p_{i}(\xi) \frac{\mathrm{d} q_{i}(\eta)}{\mathrm{d} \eta}\right)\right\}|\mathbf{J}| \mathrm{d} \xi \mathrm{d} \eta
\end{aligned}
$$


developing de squares and multiplications in (C.1) one obtains

$$
\begin{aligned}
& U_{\max }=\frac{1}{2} \int_{-1}^{1} \int_{-1}^{1}\left\{S_{1}\left(\sum_{k, h=1}^{M, N} \sum_{r, s=1}^{M, N} c_{k h} c_{r s} \frac{\mathrm{d}^{2} p_{k}(\xi)}{\mathrm{d} \xi^{2}} q_{h}(\eta) \frac{\mathrm{d}^{2} p_{r}(\xi)}{\mathrm{d} \xi^{2}} q_{\mathrm{s}}(\eta)\right)\right. \\
& +S_{2}\left(\sum_{k, h=1}^{M, N} \sum_{r, s=1}^{M, N} c_{k h} c_{r s} p_{k}(\xi) \frac{\mathrm{d}^{2} q_{h}(\eta)}{\mathrm{d} \eta^{2}} p_{r}(\xi) \frac{\mathrm{d}^{2} q_{\mathrm{s}}(\eta)}{\mathrm{d} \eta^{2}}\right) \\
& +S_{3}\left(\sum_{k, h=1}^{M, N} \sum_{r, s=1}^{M, N} c_{k h} c_{r S} \frac{\mathrm{d}^{2} p_{k}(\xi)}{\mathrm{d} \xi^{2}} q_{h}(\eta) p_{r}(\xi) \frac{\mathrm{d}^{2} q_{\mathrm{s}}(\eta)}{\mathrm{d} \eta^{2}}\right) \\
& +S_{4}\left(\sum_{k, h=1}^{M, N} \sum_{r, s=1}^{M, N} c_{k h} c_{r s} \frac{\mathrm{d} p_{k}(\xi)}{\mathrm{d} \xi} \frac{\mathrm{d} q_{h}(\eta)}{\mathrm{d} \eta} \frac{\mathrm{d} p_{r}(\xi)}{\mathrm{d} \xi} \frac{\mathrm{d} q_{r}(\eta)}{\mathrm{d} \eta}\right) \\
& +S_{5}\left(\sum_{k, h=1}^{M, N} \sum_{r, s=1}^{M, N} c_{k h} c_{r s} \frac{\mathrm{d}^{2} p_{k}(\xi)}{\mathrm{d} \xi} q_{h}(\eta) \frac{\mathrm{d} p_{r}(\xi)}{\mathrm{d} \xi} \frac{\mathrm{d} q_{\mathrm{s}}(\eta)}{\mathrm{d} \eta}\right) \\
& +S_{6}\left(\sum_{k, h=1}^{M, N} \sum_{r, s=1}^{M, N} c_{k h} c_{r s} p_{k}(\xi) \frac{\mathrm{d}^{2} q_{h}(\eta)}{\mathrm{d} \eta^{2}} \frac{\mathrm{d} p_{r}(\xi)}{\mathrm{d} \xi} \frac{\mathrm{d} q_{\mathrm{s}}(\eta)}{\mathrm{d} \eta}\right) \\
& +S_{7}\left(\sum_{k, h=1}^{M, N} \sum_{r, s=1}^{M, N} c_{k h} c_{r s} \frac{\mathrm{d}^{2} p_{k}(\xi)}{\mathrm{d} \xi^{2}} q_{h}(\eta) \frac{\mathrm{d} p_{r}(\xi)}{\mathrm{d} \xi} q_{\mathrm{s}}(\eta)\right) \\
& +S_{8}\left(\sum_{k, h=1}^{M, N} \sum_{r, s=1}^{M, N} c_{k h} c_{r s} p_{k}(\xi) \frac{\mathrm{d}^{2} q_{h}(\eta)}{\mathrm{d} \eta^{2}} p_{r}(\xi) \frac{\mathrm{d} q_{\mathrm{s}}(\eta)}{\mathrm{d} \eta}\right) \\
& +S_{9}\left(\sum_{k, h=1}^{M, N} \sum_{r, s=1}^{M, N} c_{k h} c_{r s} \frac{\mathrm{d}^{2} p_{k}(\xi)}{\mathrm{d} \xi^{2}} q_{h}(\eta) p_{r}(\xi) \frac{\mathrm{d} q_{\mathrm{s}}(\eta)}{\mathrm{d} \eta}\right) \\
& +S_{10}\left(\sum_{k, h=1}^{M, N} \sum_{r, s=1}^{M, N} c_{k h} c_{r s} p_{k}(\xi) \frac{\mathrm{d}^{2} q_{h}(\eta)}{\mathrm{d} \eta^{2}} \frac{\mathrm{d} p_{r}(\xi)}{\mathrm{d} \xi} q_{\mathrm{s}}(\eta)\right) \\
& +S_{11}\left(\sum_{k, h=1}^{M, N} \sum_{r, s=1}^{M, N} c_{k h} c_{r s} \frac{\mathrm{d} p_{k}(\xi)}{\mathrm{d} \xi} \frac{\mathrm{d} q_{h}(\eta)}{\mathrm{d} \eta} \frac{\mathrm{d} p_{r}(\xi)}{\mathrm{d} \xi} q_{\mathrm{s}}(\eta)\right) \\
& +S_{12}\left(\sum_{k, h=1}^{M, N} \sum_{r, s=1}^{M, N} c_{k h} c_{r s} \frac{\mathrm{d} p_{k}(\xi)}{\mathrm{d} \xi} \frac{\mathrm{d} q_{h}(\eta)}{\mathrm{d} \eta} p_{r}(\xi) \frac{\mathrm{d} q_{\mathrm{s}}(\eta)}{\mathrm{d} \eta}\right) \\
& +S_{13}\left(\sum_{k, h=1}^{M, N} \sum_{r, s=1}^{M, N} c_{k h} c_{r s} \frac{\mathrm{d} p_{k}(\xi)}{\mathrm{d} \xi} q_{h}(\eta) \frac{\mathrm{d} p_{r}(\xi)}{\mathrm{d} \xi} q_{\mathrm{s}}(\eta)\right) \\
& +S_{14}\left(\sum_{k, h=1}^{M, N} \sum_{r, s=1}^{M, N} c_{k h} c_{r s} p_{k}(\xi) \frac{\mathrm{d} q_{h}(\eta)}{\mathrm{d} \eta} p_{r}(\xi) \frac{\mathrm{d} q_{\mathrm{s}}(\eta)}{\mathrm{d} \eta}\right) \\
& \left.+S_{15}\left(\sum_{k, h=1}^{M, N} \sum_{r, s=1}^{M, N} c_{k h} c_{r s} \frac{\mathrm{d} p_{k}(\xi)}{\mathrm{d} \xi} q_{h}(\eta) p_{r}(\xi) \frac{\mathrm{d} q_{\mathrm{s}}(\eta)}{\mathrm{d} \eta}\right)\right\}|\mathbf{J}| \mathrm{d} \xi \mathrm{d} \eta .
\end{aligned}
$$


Then, the derivation of Eq. (C.2) with respect to each coefficient $c_{i j}, i, j=1, \ldots, N, M$ leads to

$$
\begin{aligned}
& \frac{\partial U_{\max }}{\partial c_{i j}}=\frac{1}{2} \int_{-1}^{1} \int_{-1}^{1}\left\{2 S_{1} \sum_{k, h=1}^{M, N} c_{k h} \frac{\mathrm{d}^{2} p_{i}(\xi)}{\mathrm{d} \xi^{2}} \frac{\mathrm{d}^{2} p_{k}(\xi)}{\mathrm{d} \xi^{2}} q_{j}(\eta) q_{h}(\eta)+2 S_{2} \sum_{k, h=1}^{M, N} c_{k h} p_{i}(\xi) p_{k}(\xi) \frac{\mathrm{d}^{2} q_{j}(\eta)}{\mathrm{d} \eta^{2}} \frac{\mathrm{d}^{2} q_{h}(\eta)}{\mathrm{d} \eta^{2}}\right. \\
& +S_{3} \sum_{k, h=1}^{M, N} c_{k h}\left(\frac{\mathrm{d}^{2} p_{i}(\xi)}{\mathrm{d} \xi^{2}} p_{k}(\xi) \frac{\mathrm{d}^{2} q_{j}(\eta)}{\mathrm{d} \eta^{2}} q_{h}(\eta)+\frac{\mathrm{d}^{2} p_{k}(\xi)}{\mathrm{d} \xi^{2}} p_{i}(\xi) \frac{\mathrm{d}^{2} q_{h}(\eta)}{\mathrm{d} \eta^{2}} q_{j}(\eta)\right) \\
& +2 S_{4} \sum_{k, h=1}^{M, N} c_{k h} \frac{\mathrm{d} p_{i}(\xi)}{\mathrm{d} \xi} \frac{\mathrm{d} p_{k}(\xi)}{\mathrm{d} \xi} \frac{\mathrm{d} q_{j}(\eta)}{\mathrm{d} \eta} \frac{\mathrm{d} q_{h}(\eta)}{\mathrm{d} \eta} \\
& +S_{5} \sum_{k, h=1}^{M, N} c_{k h}\left(\frac{\mathrm{d}^{2} p_{i}(\xi)}{\mathrm{d} \xi^{2}} \frac{\mathrm{d} p_{k}(\xi)}{\mathrm{d} \xi} q_{j}(\eta) \frac{\mathrm{d} q_{h}(\eta)}{\mathrm{d} \eta}+\frac{\mathrm{d}^{2} p_{k}(\xi)}{\mathrm{d} \xi^{2}} \frac{\mathrm{d} p_{i}(\xi)}{\mathrm{d} \xi} q_{h}(\eta) \frac{\mathrm{d} q_{j}(\eta)}{\mathrm{d} \eta}\right) \\
& +S_{6} \sum_{k, h=1}^{M, N} c_{k h}\left(p_{i}(\xi) \frac{\mathrm{d} p_{k}(\xi)}{\mathrm{d} \xi} \frac{\mathrm{d}^{2} q_{j}(\eta)}{\mathrm{d} \eta^{2}} \frac{\mathrm{d} q_{h}(\eta)}{\mathrm{d} \eta}+p_{k}(\xi) \frac{\mathrm{d} p_{i}(\xi)}{\mathrm{d} \xi} \frac{\mathrm{d}^{2} q_{h}(\eta)}{\mathrm{d} \eta^{2}} \frac{\mathrm{d} q_{j}(\eta)}{\mathrm{d} \eta}\right) \\
& +S_{7} \sum_{k, h=1}^{M, N} c_{k h} q_{j}(\eta) q_{h}(\eta)\left(\frac{\mathrm{d}^{2} p_{i}(\xi)}{\mathrm{d} \xi} \frac{\mathrm{d} p_{k}(\xi)}{\mathrm{d} \xi}+\frac{\mathrm{d}^{2} p_{k}(\xi)}{\mathrm{d} \xi} \frac{\mathrm{d} p_{i}(\xi)}{\mathrm{d} \xi}\right) \\
& +S_{8} \sum_{k, h=1}^{M, N} c_{k h} p_{i}(\xi) p_{k}(\xi)\left(\frac{\mathrm{d}^{2} q_{j}(\eta)}{\mathrm{d} \eta^{2}} \frac{\mathrm{d} q_{h}(\eta)}{\mathrm{d} \eta}+\frac{\mathrm{d}^{2} q_{h}(\eta)}{\mathrm{d} \eta^{2}} \frac{\mathrm{d} q_{j}(\eta)}{\mathrm{d} \eta}\right) \\
& +S_{9} \sum_{k, h=1}^{M, N} c_{k h}\left(\frac{\mathrm{d}^{2} p_{i}(\xi)}{\mathrm{d} \xi^{2}} p_{k}(\xi) q_{j}(\eta) \frac{\mathrm{d} q_{h}(\eta)}{\mathrm{d} \eta}+\frac{\mathrm{d}^{2} p_{k}(\xi)}{\mathrm{d} \xi^{2}} p_{i}(\xi) q_{h}(\eta) \frac{\mathrm{d} q_{j}(\eta)}{\mathrm{d} \eta}\right) \\
& +S_{10} \sum_{k, h=1}^{M, N} c_{k h}\left(p_{i}(\xi) \frac{\mathrm{d} p_{k}(\xi)}{\mathrm{d} \xi} \frac{\mathrm{d}^{2} q_{j}(\eta)}{\mathrm{d} \eta^{2}} q_{h}(\eta)+p_{k}(\xi) \frac{\mathrm{d} p_{i}(\xi)}{\mathrm{d} \xi} \frac{\mathrm{d}^{2} q_{h}(\eta)}{\mathrm{d} \eta^{2}} q_{j}(\eta)\right) \\
& +S_{11} \sum_{k, h=1}^{M, N} c_{k h} \frac{\mathrm{d} p_{i}(\xi)}{\mathrm{d} \xi} \frac{\mathrm{d} p_{k}(\xi)}{\mathrm{d} \xi}\left(\frac{\mathrm{d} q_{j}(\eta)}{\mathrm{d} \eta} q_{h}(\eta)+\frac{\mathrm{d} q_{h}(\eta)}{\mathrm{d} \eta} q_{j}(\eta)\right) \\
& +S_{12} \sum_{k, h=1}^{M, N} c_{k h} \frac{\mathrm{d} q_{j}(\eta)}{\mathrm{d} \eta} \frac{\mathrm{d} q_{h}(\eta)}{\mathrm{d} \eta}\left(\frac{\mathrm{d} p_{i}(\xi)}{\mathrm{d} \xi} p_{k}(\xi)+\frac{\mathrm{d} p_{k}(\xi)}{\mathrm{d} \xi} p_{i}(\xi)\right) \\
& +S_{13} 2 \sum_{k, h=1}^{M, N} c_{k h} \frac{\mathrm{d} p_{i}(\xi)}{\mathrm{d} \xi} \frac{\mathrm{d} p_{k}(\xi)}{\mathrm{d} \xi} q_{j}(\eta) q_{h}(\eta)+S_{14} 2 \sum_{k, h=1}^{M, N} c_{k h} p_{i}(\xi) p_{k}(\xi) \frac{\mathrm{d} q_{j}(\eta)}{\mathrm{d} \eta} \frac{\mathrm{d} q_{h}(\eta)}{\mathrm{d} \eta} \\
& \left.+S_{15} \sum_{k, h=1}^{M, N} c_{k h}\left(\frac{\mathrm{d} p_{i}(\xi)}{\mathrm{d} \xi} p_{k}(\xi) q_{j}(\eta) \frac{\mathrm{d} q_{h}(\eta)}{\mathrm{d} \eta}+\frac{\mathrm{d} p_{k}(\xi)}{\mathrm{d} \xi} p_{i}(\xi) q_{h}(\eta) \frac{\mathrm{d} q_{j}(\eta)}{\mathrm{d} \eta}\right)\right\}|\mathbf{J}| \mathrm{d} \xi \mathrm{d} \eta .
\end{aligned}
$$

Finally, one obtains

$$
\frac{\partial U_{\max }}{\partial c_{i j}}=\frac{1}{2}\left\{\sum_{k, h=1}^{M, N} c_{k h}\left[\sum_{m=1}^{15} P_{i j k h, m}(\xi, \eta)\right]\right\},
$$

where

$$
P_{i j k h, 1}(\xi, \eta)=\int_{-1}^{1} \int_{-1}^{1} S_{1} 2 \frac{\mathrm{d}^{2} p_{i}(\xi)}{\mathrm{d} \xi^{2}} \frac{\mathrm{d}^{2} p_{k}(\xi)}{\mathrm{d} \xi^{2}} q_{j}(\eta) q_{h}(\eta)|\mathbf{J}| \mathrm{d} \xi \mathrm{d} \eta
$$




$$
\begin{aligned}
& P_{i j k h, 2}(\xi, \eta)=\int_{-1}^{1} \int_{-1}^{1} S_{2} 2 p_{i}(\xi) p_{k}(\xi) \frac{\mathrm{d}^{2} q_{j}(\eta)}{\mathrm{d} \eta^{2}} \frac{\mathrm{d}^{2} q_{h}(\eta)}{\mathrm{d} \eta^{2}}|\mathbf{J}| \mathrm{d} \xi \mathrm{d} \eta \\
& P_{i j k h, 3}(\xi, \eta)=\int_{-1}^{1} \int_{-1}^{1} S_{3}\left[\frac{\mathrm{d}^{2} p_{i}(\xi)}{\mathrm{d} \xi^{2}} p_{k}(\xi) \frac{\mathrm{d}^{2} q_{j}(\eta)}{\mathrm{d} \eta^{2}} q_{h}(\eta)+\frac{\mathrm{d}^{2} p_{k}(\xi)}{\mathrm{d} \xi^{2}} p_{i}(\xi) \frac{\mathrm{d}^{2} q_{h}(\eta)}{\mathrm{d} \eta^{2}} q_{j}(\eta)\right]|\mathbf{J}| \mathrm{d} \xi \mathrm{d} \eta \\
& P_{i j k h, 4}(\xi, \eta)=\int_{-1}^{1} \int_{-1}^{1} S_{4} 2 \frac{\mathrm{d} p_{i}(\xi)}{\mathrm{d} \xi} \frac{\mathrm{d} p_{k}(\xi)}{\mathrm{d} \xi} \frac{\mathrm{d} q_{j}(\eta)}{\mathrm{d} \eta} \frac{\mathrm{d} q_{h}(\eta)}{\mathrm{d} \eta}|\mathbf{J}| \mathrm{d} \xi \mathrm{d} \eta \\
& P_{i j k h, 5}(\xi, \eta)=\int_{-1}^{1} \int_{-1}^{1} S_{5}\left[\frac{\mathrm{d}^{2} p_{i}(\xi)}{\mathrm{d} \xi^{2}} \frac{\mathrm{d} p_{k}(\xi)}{\mathrm{d} \xi} q_{j}(\eta) \frac{\mathrm{d} q_{h}(\eta)}{\mathrm{d} \eta}+\frac{\mathrm{d}^{2} p_{k}(\xi)}{\mathrm{d} \xi^{2}} \frac{\mathrm{d} p_{i}(\xi)}{\mathrm{d} \xi} q_{h}(\eta) \frac{\mathrm{d} q_{j}(\eta)}{\mathrm{d} \eta}\right]|\mathbf{J}| \mathrm{d} \xi \mathrm{d} \eta \\
& P_{i j k h, 6}(\xi, \eta)=\int_{-1}^{1} \int_{-1}^{1} S_{6}\left[p_{i}(\xi) \frac{\mathrm{d} p_{k}(\xi)}{\mathrm{d} \xi} \frac{\mathrm{d}^{2} q_{j}(\eta)}{\mathrm{d} \eta^{2}} \frac{\mathrm{d} q_{h}(\eta)}{\mathrm{d} \eta}+p_{k}(\xi) \frac{\mathrm{d} p_{i}(\xi)}{\mathrm{d} \xi} \frac{\mathrm{d}^{2} q_{h}(\eta)}{\mathrm{d} \eta^{2}} \frac{\mathrm{d} q_{j}(\eta)}{\mathrm{d} \eta}\right]|\mathbf{J}| \mathrm{d} \xi \mathrm{d} \eta, \\
& P_{i j k h, 7}(\xi, \eta)=\int_{-1}^{1} \int_{-1}^{1} S_{7} q_{j}(\eta) q_{h}(\eta)\left[\frac{\mathrm{d}^{2} p_{i}(\xi)}{\mathrm{d} \xi} \frac{\mathrm{d} p_{k}(\xi)}{\mathrm{d} \xi}+\frac{\mathrm{d}^{2} p_{k}(\xi)}{\mathrm{d} \xi} \frac{\mathrm{d} p_{i}(\xi)}{\mathrm{d} \xi}\right]|\mathbf{J}| \mathrm{d} \xi \mathrm{d} \eta \\
& P_{i j k h, 8}(\xi, \eta)=\int_{-1}^{1} \int_{-1}^{1} S_{8} p_{i}(\xi) p_{k}(\xi)\left[\frac{\mathrm{d}^{2} q_{j}(\eta)}{\mathrm{d} \eta^{2}} \frac{\mathrm{d} q_{h}(\eta)}{\mathrm{d} \eta}+\frac{\mathrm{d}^{2} q_{h}(\eta)}{\mathrm{d} \eta^{2}} \frac{\mathrm{d} q_{j}(\eta)}{\mathrm{d} \eta}\right]|\mathbf{J}| \mathrm{d} \xi \mathrm{d} \eta, \\
& P_{i j k h, 9}(\xi, \eta)=\int_{-1}^{1} \int_{-1}^{1} S_{9}\left[\frac{\mathrm{d}^{2} p_{i}(\xi)}{\mathrm{d} \xi^{2}} p_{k}(\xi) q_{j}(\eta) \frac{\mathrm{d} q_{h}(\eta)}{\mathrm{d} \eta}+\frac{\mathrm{d}^{2} p_{k}(\xi)}{\mathrm{d} \xi^{2}} p_{i}(\xi) q_{h}(\eta) \frac{\mathrm{d} q_{j}(\eta)}{\mathrm{d} \eta}\right]|\mathbf{J}| \mathrm{d} \xi \mathrm{d} \eta, \\
& P_{i j k h, 10}(\xi, \eta)=\int_{-1}^{1} \int_{-1}^{1} S_{10}\left[p_{i}(\xi) \frac{\mathrm{d} p_{k}(\xi)}{\mathrm{d} \xi} \frac{\mathrm{d}^{2} q_{j}(\eta)}{\mathrm{d} \eta^{2}} q_{h}(\eta)+p_{k}(\xi) \frac{\mathrm{d} p_{i}(\xi)}{\mathrm{d} \xi} \frac{\mathrm{d}^{2} q_{h}(\eta)}{\mathrm{d} \eta^{2}} q_{j}(\eta)\right]|\mathbf{J}| \mathrm{d} \xi \mathrm{d} \eta, \\
& P_{i j k h, 11}(\xi, \eta)=\int_{-1}^{1} \int_{-1}^{1} S_{11} \frac{\mathrm{d} p_{i}(\xi)}{\mathrm{d} \xi} \frac{\mathrm{d} p_{k}(\xi)}{\mathrm{d} \xi}\left[\frac{\mathrm{d} q_{j}(\eta)}{\mathrm{d} \eta} q_{h}(\eta)+\frac{\mathrm{d} q_{h}(\eta)}{\mathrm{d} \eta} q_{j}(\eta)\right]|\mathbf{J}| \mathrm{d} \xi \mathrm{d} \eta, \\
& P_{i j k h, 12}(\xi, \eta)=\int_{-1}^{1} \int_{-1}^{1} S_{12} \frac{\mathrm{d} q_{j}(\eta)}{\mathrm{d} \eta} \frac{\mathrm{d} q_{h}(\eta)}{\mathrm{d} \eta}\left[\frac{\mathrm{d} p_{i}(\xi)}{\mathrm{d} \xi} p_{k}(\xi)+\frac{\mathrm{d} p_{k}(\xi)}{\mathrm{d} \xi} p_{i}(\xi)\right]|\mathbf{J}| \mathrm{d} \xi \mathrm{d} \eta, \\
& P_{i j k h, 13}(\xi, \eta)=\int_{-1}^{1} \int_{-1}^{1} S_{13} 2 \frac{\mathrm{d} p_{i}(\xi)}{\mathrm{d} \xi} \frac{\mathrm{d} p_{k}(\xi)}{\mathrm{d} \xi} q_{j}(\eta) q_{h}(\eta)|\mathbf{J}| \mathrm{d} \xi \mathrm{d} \eta \\
& P_{i j k h, 14}(\xi, \eta)=\int_{-1}^{1} \int_{-1}^{1} S_{14} 2 p_{i}(\xi) p_{k}(\xi) \frac{\mathrm{d} q_{j}(\eta)}{\mathrm{d} \eta} \frac{\mathrm{d} q_{h}(\eta)}{\mathrm{d} \eta}|\mathbf{J}| \mathrm{d} \xi \mathrm{d} \eta \\
& P_{i j k h, 15}(\xi, \eta)=\int_{-1}^{1} \int_{-1}^{1} S_{15}\left[\frac{\mathrm{d} p_{i}(\xi)}{\mathrm{d} \xi} p_{k}(\xi) q_{j}(\eta) \frac{\mathrm{d} q_{h}(\eta)}{\mathrm{d} \eta}+\frac{\mathrm{d} p_{k}(\xi)}{\mathrm{d} \xi} p_{i}(\xi) q_{h}(\eta) \frac{\mathrm{d} q_{j}(\eta)}{\mathrm{d} \eta}\right]|\mathbf{J}| \mathrm{d} \xi \mathrm{d} \eta .
\end{aligned}
$$

In the same manner, replacing the approximating function (15) into the expression of the maximum kinetic energy (11) one obtains 


$$
\begin{aligned}
T_{\max } & =\frac{h \rho \omega^{2}}{2} \int_{-1}^{1} \int_{-1}^{1}\left(\sum_{i, j=1}^{N, M} c_{i j} p_{i}(\xi) q_{j}(\eta)\right)^{2}|\mathbf{J}| \mathrm{d} \xi \mathrm{d} \eta \\
& =\frac{h \rho \omega^{2}}{2} \int_{-1}^{1} \int_{-1}^{1}\left(\sum_{k, h=1}^{N, M} \sum_{r, s=1}^{N, M} c_{k h} c_{r s} p_{k}(\xi) q_{h}(\eta) p_{r}(\xi) q_{\mathrm{s}}(\eta)\right)|\mathbf{J}| \mathrm{d} \xi \mathrm{d} \eta .
\end{aligned}
$$

Then, the derivation of Eq. (C.5) with respect to each coefficient $c_{i j}, i, j=1, \ldots, N, M$ leads to

$$
\frac{\partial T_{\max }}{\partial c_{i j}}=\frac{\rho h}{2} \omega^{2}\left\{2 \int_{-1}^{1} \int_{-1}^{1} \sum_{k, h=1}^{N, M} c_{k h} p_{i}(\xi) p_{k}(\xi) q_{j}(\eta) q_{h}(\eta)|\mathbf{J}| \mathrm{d} \xi \mathrm{d} \eta\right\} .
$$

Finally, replacing the approximating function (15) into the expression of the potential energy (12) one obtains

$$
V=-\int_{-1}^{1} \int_{-1}^{1} q(\xi, \eta)\left(\sum_{i, j=1}^{N, M} c_{i j} p_{i}(\xi) q_{j}(\eta)\right)|\mathbf{J}| \mathrm{d} \xi \mathrm{d} \eta
$$

The derivation of Eq. (C.7) with respect to each coefficient $c_{i j}, i, j=1, \ldots, N, M$ leads to

$$
\frac{\partial V}{\partial c_{i j}}=-\left\{\int_{-1}^{1} \int_{-1}^{1} q(\xi, \eta) p_{i}(\xi) q_{j}(\eta)|\mathbf{J}| \mathrm{d} \xi \mathrm{d} \eta\right\} .
$$

Eqs. (C.4), (C.6) and (C.8) leads to the governing equations (20) and (21) established in the main text.

\section{References}

[1] S. Timoshenko, S. Woinowsky-Krieger, Theory of Plates and Shells, McGraw-Hill, New York, 1959.

[2] A.W. Leissa, Vibration of plates (NASA SP-160), Office of Technology Utilization, NASA, Washington, DC, 1969.

[3] A.W. Leissa, Recent studies in plate vibrations: 1981-1985. Part I: Classical theory, The Shock and Vibration Digest 19 (1987) $11-18$.

[4] R.D. Blevins, Formulas for natural frequency and mode shape, Krieger Publishing Company, Malabar, FL, 1993.

[5] C.W. Bert, Research on dynamics of composite and sandwich plates, The Shock and Vibration Digest (1982) 17-34.

[6] C.W. Bert, Research on dynamics behaviour of composite and sandwich plates-V: Part I, The Shock and Vibration Digest 23 (1991) 3-14.

[7] C.W. Bert, Research on dynamics behaviour of composite and sandwich plates-V: Part II, The Shock and Vibration Digest 23 (1991) 9-21.

[8] O.C. Zienkiewicz, R.L. Taylor, The Finite Element Method, fourth ed., McGraw-Hill, New York, 1991.

[9] J.N. Reddy, Finite Element Method, second ed., McGraw-Hill, New York, 1993.

[10] T.M. Hrudey, M.M. Harbok, Singularity finite elements for plate bending, J. Engrg. Mech. ASCE 112 (1986) 666-681.

[11] W.Y. Ly, Y.K. Cheung, E.G. Tham, Spline finite strip analysis of general plates, J. Engrg. Mech. ASCE 112 (1986) $43-54$.

[12] Y.K. Cheung, L.G. Tham, W.Y. Li, Free vibration and static analysis of general plate by spline finite strip, Computat. Mech. 3 (1988) 187-197.

[13] H.Y. Sheng, J.Q. Ye, A state space finite element for laminated composite plates, Comput. Methods Appl. Mech. Engrg. 191 (2002) 4259-4276.

[14] K. Rektorys, Variational Methods in Mathematics, Science and Engineering, Reidel Co., Dordrecht, 1980.

[15] R.B. Bhat, Plate deflection using orthogonal polynomials, J. Engrg. Mech. ASCE 101 (1985) 1301-1309.

[16] R.B. Bhat, Natural frequencies of rectangular plates using characteristic orthogonal polynomials in Rayleigh-Ritz method, J. Sound Vibr. 102 (1985) 493-499.

[17] K.M. Liew, K.Y. Lam, A Rayleigh-Ritz approach to transverse vibration of isotropic and anisotropic trapezoidal plates using orthogonal plate functions, Int. J. Solids Struct. 27 (1991) 189-203.

[18] K.M. Liew, Response of plates of arbitrary shape subject to static loading, J. Engrg. Mech. 118 (1992) $1783-1794$.

[19] K.M. Liew, Vibration of symmetrically laminated cantilever plates, Int. J. Mech. Sci. 34 (1992) $299-308$. 
[20] K.M. Liew, C.W. Lim, Vibratory characteristics of general laminates. I: Symmetric trapezoids, J. Sound Vibr. 183 (1995) 615-642.

[21] K.M. Liew, K.Y. Lam, S.T. Chow, Free vibration analysis of rectangular plates using orthogonal plate function, Comput. Struct. 34 (1990) 79-85.

[22] K.M. Liew, K.Y. Lam, Application of two-dimensional orthogonal plate function to flexural vibration of skew plates, J. Sound Vibr. 139 (1990) 241-252.

[23] S.T. Chow, K.M. Liew, K.Y. Lam, Transverse vibration of symmetrically laminated rectangular composite plates, Compos. Struct. 20 (1992) 213-226.

[24] K.M. Liew, Vibration of symmetrically laminated cantilever trapezoidal composite plates, Int. J. Mech. Sci. 34 (1992) $299-308$.

[25] C.W. Lim, K.M. Liew, Vibration of pretwisted cantilever trapezoidal symmetric laminates, Acta Mech. 111 (1995) 193-208.

[26] C.W. Lim, K.M. Liew, S. Kitipornchai, Vibration of arbitrarily laminated plates of general trapezoidal planform, J. Acoust. Soc. Am. 100 (1996) 3674-3685.

[27] K.M. Liew, C.W. Lim, Vibratory characteristics of pretwisted cantilever trapezoids of unsymmetric laminates, AIAA J. 34 (1996) 1041-1050.

[28] C.W. Lim, S. Kitipornchai, K.M. Liew, A free vibration analysis of doubly connected super-elliptical laminated composite plates, Compos. Sci. Technol. 58 (1998) 435-445.

[29] J.N. Reddy, Mechanics of Laminated Anisotropic Plates: Theory and Analysis, CRC Press, Boca Raton, FL, 1997.

[30] J.M. Whitney, Structural Analysis of Laminated Anisotropic Plates, Technomic Publishing Co. Inc., Pennsylvania, USA, 1987.

[31] L.G. Nallim, Mechanics of Anisotropic Plates: A Variational Approach, Doctors Thesis, National University of Salta, 2003.

[32] R.O. Grossi, L.G. Nallim, Boundary and eigenvalue problems for generally restrained anisotropic plates, J. Multi-body Dyn. 217 (2003) 241-251.

[33] L. Kantorovich, V. Krylov, Approximate Methods of Higher Analysis, Interscience Publishers, 1964.

[34] S. Mikhlin, Variational Methods of Mathematical Physics, Mac Millan Co., New York, 1964.

[35] L.G. Nallim, R.O. Grossi, On the use of orthogonal polynomials in the study of anisotropic plates, J. Sound Vibr. 264 (2003) 1201-1207.

[36] S. Wang, Vibration of thin skew fibre reinforced composite laminates, J. Sound Vibr. 201 (1997) 335-352.

[37] G. Romero, L. Alvarez, E. Alanís, L. Nallim, R. Grossi, Study of a vibrating plate: comparison between experimental (ESPI) and analytical results, Optics Lasers Engrg. 40 (2003) 81-90. 